\title{
Induced Interval-Valued Intuitionistic Fuzzy Hybrid Aggregation Operators with TOPSIS Order-Inducing Variables
}

\author{
Jun-Ling Zhang ${ }^{1}$ and Xiao-Wen $\mathbf{Q i}^{1,2,3}$ \\ ${ }^{1}$ School of Economics and Management, Zhejiang Normal University, Jinhua 321004, China \\ ${ }^{2}$ Department of Management, Hefei University of Technology, Hefei 230009, China \\ ${ }^{3}$ Key Laboratory of Process Optimization and Intelligent Decision-Making, Ministry of Education, \\ Hefei 230009, China \\ Correspondence should be addressed to Xiao-Wen Qi, springoasis_qi@163.com
}

Received 1 December 2011; Revised 24 February 2012; Accepted 14 March 2012

Academic Editor: Huijun Gao

Copyright (C) 2012 J.-L. Zhang and X.-W. Qi. This is an open access article distributed under the Creative Commons Attribution License, which permits unrestricted use, distribution, and reproduction in any medium, provided the original work is properly cited.

\begin{abstract}
Two induced aggregation operators with novelly designed TOPSIS order-inducing variables are proposed: Induced Interval-valued Intuitionistic Fuzzy Hybrid Averaging (I-IIFHA) operator and Induced Interval-valued Intuitionistic Fuzzy Hybrid Geometric (I-IIFHG) operator. The merit of two aggregation operators is that they can consider additional preference information of decision maker's attitudinal characteristics besides argument-dependent information and argumentindependent information. Some desirable properties of I-IIFHA and I-IIFHG are studied and theoretical analysis also shows that they can include a wide range of aggregation operators as special cases. Further, we extend these operators to form a novel group decision-making method for selecting the most desirable alternative in multiple attribute multi-interest group decisionmaking problems with attribute values and decision maker's interest values taking the form of interval-valued intuitionistic fuzzy numbers, and application research to real estate purchase selection shows its practicality.
\end{abstract}

\section{Introduction}

In many practical decision-making activities, the available knowledge of decision makers is vague or imprecise and cannot be assessed with numerical values, so Zadeh's fuzzy set theory [1] and Atanassov's intuitionistic fuzzy set (IFS) theory [2] are introduced to deal with uncertain decision environments. Over the last decades, the increasing researches have indicated that Atanassov's IFS and interval-valued intuitionistic fuzzy set (IVIFS) [3] characterized by a membership function and nonmembership function are more suitable to deal with fuzziness and uncertainty than the generalization of fuzzy set developed by Zadeh. 
So recently, particular aggregation operators and corresponding decision-making methods have been studied and widely used for solving different decision-making problems under intuitionistic fuzzy or interval-valued intuitionistic fuzzy environments. As for the studies on single-person multiattribute decision-making (MADM) problems, $\mathrm{Xu}$ [4] investigated the methodology for solving multiattribute decision-making problems with intuitionistic fuzzy preference information aggregated by the intuitionistic fuzzy-weighted averaging (IFWA) operator, the intuitionistic fuzzy-ordered weighted averaging (IFOWA) operator, and the intuitionistic fuzzy hybrid averaging (IFHA) operator. Xu and Yager [5] proposed some geometric aggregation operators based on intuitionistic fuzzy sets, such as the intuitionistic fuzzy-weighted geometric (IFWG) operator, the intuitionistic fuzzy-ordered weighted geometric (IFOWG) operator and the intuitionistic fuzzy hybrid geometric (IFHG) operator and then applied them to multiple attribute decision-making. $\mathrm{Xu}$ and Xia [6] studied a method for multiattribute decision-making under IFS environment with induced generalized aggregation operators (I-GIFCOA and BS I-GIFCOA) designed by introducing Choquet integral and Dempster-Shafer evidence theory. Tan and Chen [7] considered interactions phenomena among the decision-making criteria under IFS environment and also introduced Choquet integral to design an intuitionistic fuzzy operator (I-IFC) for multicriteria decision-making, and so forth. With respect to the researches on dynamic multiattribute decision-making (DMADM) problems, $\mathrm{Xu}$ and Yager [8] developed two new dynamic intuitionistic fuzzy aggregation operators to solve the dynamic intuitionistic fuzzy multi-attribute decision-making problems. Wei [9] proposed the dynamic intuitionistic fuzzy-weighted geometric (DIFWG) operator and developed a procedure based on the DIFWG and IFWG operators to solve dynamic intuitionistic fuzzy multi-attribute decisionmaking (DIF-MADM) problems where all the decision information about attribute values takes the form of intuitionistic fuzzy numbers collected at different periods. For the researches on multiple attribute group decision-making (MAGDM) problems, $\mathrm{Xu}$ and Chen $[10,11]$ investigated decision-making methods for the IVIFS environment and defined several arithmetic aggregation operators and geometric aggregation operators, such as the interval-valued intuitionistic fuzzy-weighted averaging (IIFWA) operator, the intervalvalued intuitionistic fuzzy-ordered weighted averaging (IIFOWA) operator, the intervalvalued intuitionistic fuzzy hybrid averaging (IIFHA) operator, interval-valued intuitionistic fuzzy geometric (IIFG) operator, and interval-valued intuitionistic fuzzy-weighted geometric (IIFWG) operator. Wei [12] developed some induced geometric aggregation operators based on IFS and IVIFS, such as the induced intuitionistic fuzzy-ordered weighted geometric (I-IFOWG) operator and induced interval-valued intuitionistic fuzzy-ordered weighted geometric (I-IIFOWG) operator, and also extended them to solve the multi-attribute decisionmaking problems, and so forth.

As can be seen from the researches mentioned, aggregation operators for fusion of decision preference information play a central role in solving different kinds of decisionmaking problems. In the existing literatures, different types of aggregation operators and their extensions can be found for fusing intuitionistic fuzzy and interval-valued intuitionistic fuzzy information, such as the weighted aggregation operators IFWA, IFWG, IIFWA, and IIFG, the ordered weighted aggregation operators IFOWA, IFOWG, IIFOWA, and IIFWG; the induced aggregation operators I-GIFCOA, I-GIFCOA, I-IFC, I-IFOWG, and I-IIFOWG; the hybrid aggregation operators IFHA, IFHG, and IIFHA. From the aggregation process of these representative operators, it can be seen that weighted aggregation operators or ordered weighted aggregation operators can manage to utilize argument-dependent (value weights) or argument-independent (position weights) decision preference information [4, 
13-17]; hybrid operators combine the advantages of both ordered weighted aggregation and weighted aggregation operators and manage to consider both position weights and value weights [17-23]; induced operators [24-32] and generalized induced operators [6, 31, 33-38] integrating order-inducing variables are able to consider additional reordering criteria that do not depend on the values of the arguments. Obviously the combination of hybrid operators and induced operators can enable the aggregation process to utilize all the argumentdependent preference information, argument-independent preference information and additional preference criteria, and so as to select the most desirable alternative in accordance with preference of decision makers. Such as, Merigó and Casanovas [20] presented an induced generalized hybrid averaging (IGHA) operator under the exact numerical environment by combination of the induced aggregation operator and the hybrid aggregation operator. But there is scarcely any research on the induced hybrid aggregation operators for the intuitionistic fuzzy or interval-valued intuitionistic fuzzy decision environment as far as can be seen from existing literatures.

In this paper, we investigate the induced hybrid operators with order-inducing variables not directly given by decision makers and their application to multiple attribute group decision-making with multi-interests under the interval-valued intuitionistic fuzzy decision environment. We present two induced interval-valued intuitionistic fuzzy hybrid aggregation operators: Induced Interval-valued Intuitionistic Fuzzy Hybrid Averaging (IIIFHA) operator and Induced Interval-valued Intuitionistic Fuzzy Hybrid Geometric (IIIFHG) operator. The two operators manage to use information more completely through two aspects: (1) hybridly including argument-dependent weighting vector and argumentindependent weighting vector under interval-valued intuitionistic fuzzy environment, and (2) extending the IIFHA and IIFHG operators by including order-inducing variables ensured with a designed TOPSIS-based method in the aggregation process to use more attitudinal information in accordance with different decision maker's interests.

The first main advantage of this paper is that the proposed I-IIFHA and I-IIFHG operators can help decision-making process to consider preference information in a more complete way during aggregation than other ordinary aggregation operators mentioned previously. And obviously, if the order-inducing variables are the argument ordering, then the proposed induced operators can be reduced to other ordinary aggregation operators. So the I-IIFHA and I-IIFHG operators can include a wide range of interval-valued intuitionistic fuzzy aggregation operators as special cases, such as IIFHA, IIFOWA, IIFWA, IIFHG, IIFOWG and IIFWG operators. The other important contribution of this paper is the proposal of using the information related to various decision makers' interests to deduce orderinducing criteria in induced interval-valued intuitionistic fuzzy hybrid aggregation. Recently, although many researches have been conducted on various induced operators, there are few researches on the methods to ensure order-inducing variables. Order-inducing variables as the reordering step can enable induced aggregation operators to consider additional decision rules that do not depend on the values of the arguments, such as attitudinal characteristic information of decision makers. In the literature [27, 39, 40], Merigó and Casanovas introduced some induced aggregation operators under exact number decision environment with a complex reordering process by using Euclidean or Hamming distance measures and the man-provided order-inducing variables according to the experts' interests. But sometimes, when decision makers cannot provide their exact preference directly because of time pressure or limited expertise related to the problem domain, certain methods should be developed to ensure order-inducing variables. Marin et al. [25] proposed to use the specificity and fuzziness measures of the unbalanced linguistic terms to induce the 
order-inducing variables of the arguments. For the two situations with or without manprovided additional preference, Chiclana et al. [24] presented three induced aggregation operators under exact number environment: Importance IOWA, consistency IOWA, and preference IOWA operator; the former two operators are based on the reliability of the given arguments; the latter operator is based on a relative preference associated with each one of given arguments. In this paper, we also consider the situation where the decision makers provide their own interests, but the difference is that the decision makers express their attitudinal characteristics in the form of interval-valued intuitionistic fuzzy values which can represent the information in a more complete way, and meanwhile, they can provide both ideal solutions and negative solutions. To do this, in I-IIFHA and I-IIFHG we introduce the TOPSIS method to induce the order-inducing variables, which is called TOPSIS order-inducing variables. The developed TOPSIS order-inducing method especially can also be used to induce the order-inducing variables in the situation of more than one ideal solution or negative solution provided and in the situation of no ideal solution or negative solution provided. We can use the proposed induced hybrid aggregation operators with TOPSIS order-inducing variables to utilize preference information more completely under interval-valued intuitionistic fuzzy decision environment for solving multiple attribute group decision-making problems with multi-interests.

The remainder of this paper is organized as follows. In Section 2, we give a review of basic concepts related to intuitionistic fuzzy sets and interval-valued intuitionistic fuzzy sets, and overview some aggregation operators. In Section 3, we propose induced intervalvalued intuitionistic fuzzy hybrid averaging (I-IIFHA) operator and induced intervalvalued intuitionistic fuzzy hybrid geometric (I-IIFHG) operator and analyze some desirable properties; then we introduce TOPSIS method to ensure order-inducing variables that reflect the complex attitudinal characteristic of the decision maker. In Section 4, based on the I-IIFHA and I-IIFHG operators, an approach is developed to solve the multiple attribute decisionmaking problems under the interval-valued intuitionistic fuzzy environment. In Section 5, an example is given to illustrate validity and practicality of the proposed methods. Finally, we conclude the paper.

\section{Preliminaries}

\subsection{Related Definitions of IVIFSs}

Some basic concepts on IVIFSs are introduced here in after to facilitate future discussions.

In 1986, Atanassov [2] generalized the concept of fuzzy set and defined the concept of intuitionistic fuzzy set as follows.

Definition 2.1 (see [2]). A generalized fuzzy set called intuitionistic fuzzy set is shown as follows:

$$
A=\left\{\left\langle x_{i}, \mu_{A}\left(x_{i}\right), v_{A}\left(x_{i}\right)\right\rangle \mid x_{i} \in X\right\}
$$

in which $\mu_{A}$ means a membership function, and $v_{A}$ means a nonmembership, with the condition $0 \leq \mu_{A}\left(x_{i}\right)+v_{A}\left(x_{i}\right) \leq 1, \mu_{A}\left(x_{i}\right)$, and $v_{A}\left(x_{i}\right) \in[0,1]$, for all $x_{i} \in X . A=\mu_{A}\left(x_{i}\right)=v_{A}\left(x_{i}\right)$ especially; the given IFS $A$ is degraded to an ordinary fuzzy set. 
In reality, it may not be easy to identify exact values for the membership and nonmembership degrees of an element to a set. In this case, a range of values may be a more appropriate measurement to accommodate the vagueness. As such, Atanassov and Gargov [3] introduce the notion of interval-valued intuitionistic fuzzy set (IVIFS).

Definition 2.2 (see [3]). An interval-valued intuitionistic fuzzy set (IVIFS) $\tilde{A}$ in $X$ is defined by

$$
\tilde{A}=\left\{\left\langle x_{i}, \tilde{\mu}_{\tilde{A}}\left(x_{i}\right), \tilde{v}_{\tilde{A}}\left(x_{i}\right)\right\rangle \mid x_{i} \in X\right\}=\left\{\left\langle x_{i},\left[\mu_{\tilde{A}}^{L}\left(x_{i}\right), \mu_{\widetilde{A}}^{U}\left(x_{i}\right)\right],\left[v_{\tilde{A}}^{L}\left(x_{i}\right), v_{\widetilde{A}}^{U}\left(x_{i}\right)\right]\right\rangle \mid x_{i} \in X\right\},
$$

where $0 \leq \mu_{\tilde{A}}^{L}\left(x_{i}\right) \leq \mu_{\tilde{A}}^{U}\left(x_{i}\right) \leq 1,0 \leq v_{\tilde{A}}^{L}\left(x_{i}\right) \leq v_{\tilde{A}}^{U}\left(x_{i}\right) \leq 1,0 \leq \mu_{\tilde{A}}^{U}\left(x_{i}\right)+v_{\tilde{A}}^{U}\left(x_{i}\right) \leq 1$ for all $x_{i} \in X$. Similarity, the intervals $\tilde{\mu}_{\tilde{A}}\left(x_{i}\right)$ and $\tilde{v}_{\tilde{A}}\left(x_{i}\right)$ denote the degree of membership and nonmembership of an element to a set.

Then, we introduce some formulae to calculate the distance of IVIFSs.

Definition 2.3. Suppose that two interval-valued intuitionistic fuzzy sets (IVIFSs) $\widetilde{A}$ and $\widetilde{B}$ in $\mathrm{X}$ are defined as

$$
\begin{aligned}
& \tilde{A}=\left\{\left\langle x_{i}, \tilde{\mu}_{\widetilde{A}}\left(x_{i}\right), \tilde{v}_{\widetilde{A}}\left(x_{i}\right)\right\rangle \mid x_{i} \in X\right\}=\left\{\left\langle x_{i},\left[\mu_{\tilde{A}}^{L}\left(x_{i}\right), \mu_{\widetilde{A}}^{U}\left(x_{i}\right)\right],\left[v_{\widetilde{A}}^{L}\left(x_{i}\right), v_{\widetilde{A}}^{U}\left(x_{i}\right)\right]\right\rangle \mid x_{i} \in X\right\}, \\
& \widetilde{B}=\left\{\left\langle x_{i}, \tilde{\mu}_{\widetilde{B}}\left(x_{i}\right), \tilde{v}_{\widetilde{B}}\left(x_{i}\right)\right\rangle \mid x_{i} \in X\right\}=\left\{\left\langle x_{i},\left[\mu_{\tilde{B}}^{L}\left(x_{i}\right), \mu_{\widetilde{B}}^{U}\left(x_{i}\right)\right],\left[v_{\widetilde{B}}^{L}\left(x_{i}\right), v_{\widetilde{B}}^{U}\left(x_{i}\right)\right]\right\rangle \mid x_{i} \in X\right\} .
\end{aligned}
$$

The normalized Euclidean distance measure is

$$
\begin{aligned}
& D_{1}(\tilde{A}, \tilde{B})= \\
& \sqrt{\frac{1}{6 n} \sum_{i=1}^{n}\left[\left(\mu_{\tilde{A}}^{L}\left(x_{i}\right)-\mu_{\tilde{B}}^{L}\left(x_{i}\right)\right)^{2}+\left(\mu_{\tilde{A}}^{U}\left(x_{i}\right)-\mu_{\tilde{B}}^{U}\left(x_{i}\right)\right)^{2}+\left(v_{\tilde{A}}^{L}\left(x_{i}\right)-v_{\tilde{B}}^{L}\left(x_{i}\right)\right)^{2}+\mathcal{B}+\mathscr{H}+\mathcal{K}\right]},
\end{aligned}
$$

where $\mathcal{B}$ denotes $\left(v_{\tilde{A}}^{U}\left(x_{i}\right)-v_{\tilde{B}}^{U}\left(x_{i}\right)\right)^{2}$, $\mathscr{t}$ denotes $\left(\pi_{\tilde{A}}^{L}\left(x_{i}\right)-\pi_{\tilde{B}}^{L}\left(x_{i}\right)\right)^{2}$, and $\mathcal{K} \operatorname{denotes}\left(\pi_{\tilde{A}}^{U}\left(x_{i}\right)-\right.$ $\left.\pi_{\widetilde{B}}^{U}\left(x_{i}\right)\right)^{2}$.

The normalized Hamming distance measure is

$$
\begin{aligned}
& D_{2}(\tilde{A}, \widetilde{B})= \\
& \frac{1}{6 n} \sum_{i=1}^{n}\left|\mu_{\tilde{A}}^{L}\left(x_{i}\right)-\mu_{\tilde{B}}^{L}\left(x_{i}\right)\right|+\left|\mu_{\tilde{A}}^{U}\left(x_{i}\right)-\mu_{\tilde{B}}^{U}\left(x_{i}\right)\right|+\left|v_{\tilde{A}}^{L}\left(x_{i}\right)-v_{\tilde{B}}^{L}\left(x_{i}\right)\right|+\left|v_{\tilde{A}}^{U}\left(x_{i}\right)-v_{\widetilde{B}}^{U}\left(x_{i}\right)\right| \\
& \quad+\left|\pi_{\tilde{A}}^{L}\left(x_{i}\right)-\pi_{\tilde{B}}^{L}\left(x_{i}\right)\right|+\left|\pi_{\tilde{A}}^{U}\left(x_{i}\right)-\pi_{\tilde{B}}^{U}\left(x_{i}\right)\right| .
\end{aligned}
$$


The normalized Hausdorff distance measure is

$$
\begin{aligned}
& D_{3}(\tilde{A}, \tilde{B})= \\
& \frac{1}{n} \sum_{i=1}^{n} \max \left\{\left|\mu_{\tilde{A}}^{L}\left(x_{i}\right)-\mu_{\tilde{B}}^{L}\left(x_{i}\right)\right|,\left|\mu_{\tilde{A}}^{U}\left(x_{i}\right)-\mu_{\tilde{B}}^{U}\left(x_{i}\right)\right|,\left|v_{\widetilde{A}}^{L}\left(x_{i}\right)-v_{\tilde{B}}^{L}\left(x_{i}\right)\right|,\right. \\
& \left.\quad\left|\nu_{\tilde{A}}^{U}\left(x_{i}\right)-v_{\tilde{B}}^{U}\left(x_{i}\right)\right|,\left|\pi_{\tilde{A}}^{L}\left(x_{i}\right)-\pi_{\tilde{B}}^{L}\left(x_{i}\right)\right|,\left|\pi_{\tilde{A}}^{U}\left(x_{i}\right)-\pi_{\tilde{B}}^{U}\left(x_{i}\right)\right|\right\} .
\end{aligned}
$$

In order to aggregate interval-valued intuitionistic fuzzy information, we introduce the following relations and operations given by $\mathrm{Xu}[10]$.

Definition 2.4 (see $[10])$. Let $\tilde{\alpha}=([a, b],[c, d]), \tilde{\alpha}_{1}=\left(\left[a_{1}, b_{1}\right],\left[c_{1}, d_{1}\right]\right), \tilde{\alpha}_{2}=\left(\left[a_{2}, b_{2}\right],\left[c_{2}, d_{2}\right]\right)$ be three IVIFNs; then

(1) $\tilde{\alpha}_{1} \oplus \widetilde{\alpha}_{2}=\left(\left[a_{1}+a_{2}-a_{1} a_{2}, b_{1}+b_{2}-b_{1} b_{2}\right],\left[c_{1} c_{2}, d_{1} d_{2}\right]\right)$;

(2) $\tilde{\alpha}_{1} \otimes \tilde{\alpha}_{2}=\left(\left[a_{1} a_{2}, b_{1} b_{2}\right],\left[c_{1}+c_{2}-c_{1} c_{2}, d_{1}+d_{2}-d_{1} d_{2}\right]\right)$;

(3) $\lambda \tilde{\alpha}=\left(\left[1-(1-a)^{\lambda}, 1-(1-b)^{\lambda}\right],\left[c^{\curlywedge}, d^{\lambda}\right]\right)$;

(4) $\tilde{\alpha}^{\lambda}=\left(\left[a^{\lambda}, b^{\lambda}\right],\left[1-(1-c)^{\lambda}, 1-(1-d)^{\lambda}\right]\right)$.

Definition 2.5 (see $[10])$. Let $\tilde{\alpha}=([a, b],[c, d]), \tilde{\alpha}_{1}=\left(\left[a_{1}, b_{1}\right],\left[c_{1}, d_{1}\right]\right), \tilde{\alpha}_{2}=\left(\left[a_{2}, b_{2}\right],\left[c_{2}, d_{2}\right]\right)$ be three IVIFNs, $\lambda, \lambda_{1}, \lambda_{2} \geq 0$; then

(1) $\tilde{\alpha}_{1} \oplus \tilde{\alpha}_{2}=\tilde{\alpha}_{2} \oplus \tilde{\alpha}_{1}$

(2) $\tilde{\alpha}_{1} \otimes \widetilde{\alpha}_{2}=\widetilde{\alpha}_{2} \otimes \widetilde{\alpha}_{1}$

(3) $\lambda\left(\tilde{\alpha}_{1} \oplus \tilde{\alpha}_{2}\right)=\lambda \tilde{\alpha}_{2} \oplus \lambda \tilde{\alpha}_{1}$;

(4) $\left(\tilde{\alpha}_{1} \otimes \tilde{\alpha}_{2}\right)^{\lambda}=\tilde{\alpha}_{2}^{\lambda} \otimes \tilde{\alpha}_{1}^{\curlywedge}$;

(5) $\lambda_{1} \tilde{\alpha} \oplus \lambda_{2} \tilde{\alpha}=\left(\lambda_{1}+\lambda_{2}\right) \tilde{\alpha}$;

(6) $\tilde{\alpha}^{\lambda_{1}} \otimes \tilde{\alpha}^{\lambda_{2}}=(\tilde{\alpha})^{\lambda_{1}+\lambda_{2}}$.

In order to rank alternatives, it is necessary to consider how to compare two IVIFNs. $\mathrm{Xu}$ [10] also devised an approach to compare two IVIFNs based on the concepts of score function and accuracy function.

Definition 2.6 (see [10]). For any three IVIFNs $\tilde{\alpha}=\left(\left[\mu^{L}, \mu^{U}\right],\left[v^{L}, v^{U}\right]\right), \widetilde{\alpha}_{1}=\left(\left[\mu_{1}^{L}, \mu_{1}^{U}\right]\right.$, $\left.\left[v_{1}^{L}, v_{1}^{U}\right]\right), \widetilde{\alpha}_{2}=\left(\left[\mu_{2}^{L}, \mu_{2}^{U}\right],\left[v_{2}^{L}, v_{2}^{U}\right]\right)$, score function defined as $s(\widetilde{\alpha})=(1 / 2)\left(\mu^{L}+\mu^{U}-v^{L}-v^{U}\right)$, and accuracy function defined as $h(\tilde{\alpha})=(1 / 2)\left(\mu^{L}+\mu^{U}+v^{L}+v^{U}\right)$.

If $s\left(\tilde{\alpha}_{1}\right)<s\left(\tilde{\alpha}_{2}\right)$, then $\tilde{\alpha}_{1}$ is smaller than $\tilde{\alpha}_{2}$, denoted by $\tilde{\alpha}_{1}<\tilde{\alpha}_{2}$.

If $s\left(\widetilde{\alpha}_{1}\right)=s\left(\widetilde{\alpha}_{2}\right)$, then one has the following.

If $h\left(\tilde{\alpha}_{1}\right)<h\left(\tilde{\alpha}_{2}\right)$, then $\tilde{\alpha}_{1}$ is smaller than $\tilde{\alpha}_{2}$, denoted by $\tilde{\alpha}_{1}<\tilde{\alpha}_{2}$.

If $h\left(\tilde{\alpha}_{1}\right)=h\left(\tilde{\alpha}_{2}\right)$, then $\tilde{\alpha}_{1}$ and $\tilde{\alpha}_{2}$ represent the same information, denoted by $\tilde{\alpha}_{1}=$ $\tilde{\alpha}_{2}$. 


\subsection{Some Interval-Valued Intuitionistic Fuzzy Aggregation Operators}

We first introduce definitions of interval-valued intuitionistic fuzzy-weighted averaging (IIFWA) and interval-valued intuitionistic fuzzy-weighted geometric (IIFWG) operators.

Definition 2.7 (see [10]). Let $\tilde{\alpha}_{1}, \tilde{\alpha}_{2}, \ldots, \tilde{\alpha}_{n}$ be a collection of interval arguments, where $\tilde{\alpha}_{j}=$ $\left(\left[a_{j}, b_{j}\right],\left[c_{j}, d_{j}\right]\right)$, and IIFWA operator of dimension $n$ is a mapping IIFWA: $R^{n} \rightarrow R$, that has an associated vector $w=\left(w_{1}, w_{2}, \ldots, w_{n}\right)^{T}$ such that $\sum_{j=1}^{n} w_{j}=1$ and $w_{j} \in[0,1]$; then

$$
\begin{aligned}
& \operatorname{IIFWA}_{\omega}\left(\tilde{\alpha}_{1}, \tilde{\alpha}_{2}, \ldots, \tilde{\alpha}_{n}\right)=\omega_{1} \tilde{\alpha}_{1} \oplus \omega_{2} \tilde{\alpha}_{2} \oplus \cdots \oplus \omega_{n} \tilde{\alpha}_{n} \\
& =\left(\left[1-\prod_{j=1}^{n}\left(1-a_{j}\right)^{\omega_{j}}, 1-\prod_{j=1}^{n}\left(1-b_{j}\right)^{\omega_{j}}\right],\left[\prod_{j=1}^{n} c_{j} \omega_{j}, \prod_{j=1}^{n} d_{j} \omega_{j}\right]\right) .
\end{aligned}
$$

Definition 2.8 (see [10]). Let $\tilde{\alpha}_{1}, \tilde{\alpha}_{2}, \ldots, \tilde{\alpha}_{n}$ be a collection of interval arguments, where $\tilde{\alpha}_{j}=$ $\left(\left[a_{j}, b_{j}\right],\left[c_{j}, d_{j}\right]\right)$, and IIFWG operator of dimension $n$ is a mapping IIFWG: $R^{n} \rightarrow R$, that has an associated weight vector $\omega=\left(\omega_{1}, \omega_{2}, \ldots, \omega_{n}\right)^{T}$ such that $\sum_{j=1}^{n} \omega_{j}=1$ and $\omega_{j} \in[0,1]$; then

$$
\begin{aligned}
& \operatorname{IIFWG}_{\omega}\left(\tilde{\alpha}_{1}, \tilde{\alpha}_{2}, \ldots, \widetilde{\alpha}_{n}\right)=\tilde{\alpha}_{1} \omega_{1} \otimes \widetilde{\alpha}_{2} \omega_{2} \otimes \cdots \otimes \widetilde{\alpha}_{n}{ }^{\omega_{n}} \\
& =\left(\left[\prod_{j=1}^{n} a_{j} \omega_{j}, \prod_{j=1}^{n} b_{j}{ }^{\omega_{j}}\right],\left[1-\prod_{j=1}^{n}\left(1-c_{j}\right)^{\omega_{j}}, 1-\prod_{j=1}^{n}\left(1-d_{j}\right)^{\omega_{j}}\right]\right) .
\end{aligned}
$$

Furthermore, consider the ordered positions of the interval-valued intuitionistic fuzzy values instead of weighting the interval-valued intuitionistic fuzzy values themselves. Xu and Chen [11] also proposed interval-valued intuitionistic fuzzy-ordered weighted averaging (IIFOWA) and interval-valued intuitionistic fuzzy-ordered weighted geometric (IIFOWG) operators.

Definition 2.9 (see [11]). Let $\widetilde{\alpha}_{1}, \widetilde{\alpha}_{2}, \ldots, \widetilde{\alpha}_{n}$ be a collection of interval arguments, where $\tilde{\alpha}_{j}=$ $\left(\left[a_{j}, b_{j}\right],\left[c_{j}, d_{j}\right]\right)$, and IIFOWA operator of dimension $n$ is a mapping IIFOWA: $R^{n} \rightarrow R$, that has an associated weight vector $\omega=\left(\omega_{1}, \omega_{2}, \ldots, \omega_{n}\right)^{T}$ such that $\sum_{j=1}^{n} \omega_{j}=1$ and $\omega_{j} \in[0,1]$; then

$$
\begin{aligned}
\operatorname{IIFOWA}_{\omega}\left(\widetilde{\alpha}_{1}, \tilde{\alpha}_{2}, \ldots, \widetilde{\alpha}_{n}\right)=\omega_{1} \widetilde{\alpha}_{\pi(1)} \oplus \omega_{2} \widetilde{\alpha}_{\pi(2)} \oplus \cdots \oplus \omega_{n} \widetilde{\alpha}_{\pi(n)} \\
=\left(\left[1-\prod_{j=1}^{n}\left(1-a_{\pi(j)}\right)^{\omega_{j}}, 1-\prod_{j=1}^{n}\left(1-b_{\pi(j)}\right)^{\omega_{j}}\right],\left[\prod_{j=1}^{n} c_{\pi(j)} \omega_{j}, \prod_{j=1}^{n} d_{\pi(j)} \omega_{j}\right]\right),
\end{aligned}
$$

where $\tilde{\alpha}_{\pi(1)}, \tilde{\alpha}_{\pi(2)}, \ldots, \tilde{\alpha}_{\pi(n)}$ is a permutation of $\tilde{\alpha}_{1}, \tilde{\alpha}_{2}, \ldots, \tilde{\alpha}_{n}$, such that $\tilde{\alpha}_{\pi(j-1)} \geq \tilde{\alpha}_{\pi(j)}$ for all $j$.

Definition 2.10 (see [11]). Let $\tilde{\alpha}_{1}, \tilde{\alpha}_{2}, \ldots, \widetilde{\alpha}_{n}$ be a collection of interval arguments, where $\tilde{\alpha}_{j}=$ $\left(\left[a_{j}, b_{j}\right],\left[c_{j}, d_{j}\right]\right)$, and IIFOWG operator of dimension $n$ is a mapping IIFOWG: $R^{n} \rightarrow R$, that 
has an associated weight vector $\omega=\left(\omega_{1}, \omega_{2}, \ldots, \omega_{n}\right)^{T}$ such that $\sum_{j=1}^{n} \omega_{j}=1$ and $\omega_{j} \in[0,1]$; then

$$
\begin{aligned}
& \operatorname{IIFOWG}_{\omega}\left(\tilde{\alpha}_{1}, \tilde{\alpha}_{2}, \ldots, \tilde{\alpha}_{n}\right)=\tilde{\alpha}_{\pi(1)} \omega_{1} \otimes \tilde{\alpha}_{\pi(2)} \omega_{2} \otimes \cdots \otimes \tilde{\alpha}_{\pi(n)} \omega_{n} \\
& \quad=\left(\left[\prod_{j=1}^{n} a_{\pi(j)} \omega_{j}, \prod_{j=1}^{n} b_{\pi(j)} \omega_{j}\right],\left[1-\prod_{j=1}^{n}\left(1-c_{\pi(j)}\right)^{\omega_{j}}, 1-\prod_{j=1}^{n}\left(1-d_{\pi(j)}\right)^{\omega_{j}}\right]\right),
\end{aligned}
$$

where $\tilde{\alpha}_{\pi(1)}, \tilde{\alpha}_{\pi(2)}, \ldots, \tilde{\alpha}_{\pi(n)}$ is a permutation of $\widetilde{\alpha}_{1}, \widetilde{\alpha}_{2}, \ldots, \widetilde{\alpha}_{n}$, such that $\widetilde{\alpha}_{\pi(j-1)} \geq \tilde{\alpha}_{\pi(j)}$ for all $j$.

Ordered weighted aggregation operators only consider the position weights of the interval-valued intuitionistic fuzzy values themselves. To overcome this limitation, $\mathrm{Xu}$ and Chen [11] provided definitions of interval-valued intuitionistic fuzzy hybrid averaging (IIFHA) operator and interval-valued intuitionistic fuzzy hybrid geometric (IIFHG) operator, which weights both the given interval-valued intuitionistic fuzzy value and its ordered position information.

Definition 2.11 (see [11]). An IIFHA operator of dimension $n$ is a mapping IIFHA: $R^{n} \rightarrow R$ that has an associated vector $w=\left(w_{1}, w_{2}, \ldots, w_{n}\right)^{T}$ such that $\sum_{j=1}^{n} w_{j}=1$ and $w_{j} \in[0,1]$; then

$$
\operatorname{IIFHA}_{\omega, w}\left(\widetilde{\alpha}_{1}, \tilde{\alpha}_{2}, \ldots, \widetilde{\alpha}_{n}\right)=w_{1} \dot{\tilde{\alpha}}_{\pi(1)} \oplus w_{2} \dot{\tilde{\alpha}}_{\pi(2)} \oplus \cdots \oplus w_{n} \dot{\tilde{\alpha}}_{\pi(n)}
$$

where $\dot{\tilde{\alpha}}_{\pi(j)}$ is the $j$ th largest of the weighted intuitionistic fuzzy values $\dot{\tilde{\alpha}}_{j}=n \omega_{j} \tilde{\alpha}_{j}, \omega=$ $\left(\omega_{1}, \omega_{2}, \ldots, \omega_{n}\right)^{T}$ is the weight vector $\tilde{\alpha}_{j}(j=1,2, \ldots, n)$ with $\sum_{j=1}^{n} \omega_{j}=1$ and $\omega_{j} \in[0,1]$, and $n$ is the balancing coefficient.

Definition 2.12 (see [11]). An IIFHG operator of dimension $n$ is a mapping IIFHG: $R^{n} \rightarrow R$ that has an associated vector $w=\left(w_{1}, w_{2}, \ldots, w_{n}\right)^{T}$ such that $\sum_{j=1}^{n} w_{j}=1$ and $w_{j} \in[0,1]$; then

$$
\operatorname{IIFHG}_{\omega, w}\left(\tilde{\alpha}_{1}, \tilde{\alpha}_{2}, \ldots, \tilde{\alpha}_{n}\right)=\dot{\tilde{\alpha}}_{\pi(1)} w_{1} \otimes \dot{\tilde{\alpha}}_{\pi(2)} w_{2} \otimes \cdots \otimes \dot{\tilde{\alpha}}_{\pi(n)} w_{n},
$$

where $\dot{\tilde{\alpha}}_{\pi(j)}$ is the $j$ th largest of the weighted intuitionistic fuzzy values $\dot{\tilde{\alpha}}_{j}=\tilde{\alpha}_{j}{ }^{n \omega_{j}}, \omega=\left(\omega_{1}\right.$, $\left.\omega_{2}, \ldots, \omega_{n}\right)^{T}$ is the weight vector $\tilde{\alpha}_{j}(j=1,2, \ldots, n)$ with $\sum_{j=1}^{n} \omega_{j}=1$ and $\omega_{j} \in[0,1]$, and $n$ is the balancing coefficient.

The induced operator is an extension of the ordinary aggregation operators. The main difference is that the reordering step is not carried out with the arguments values. In this case, the reordering step is developed with order-inducing variables that reflect a more complex reordering process. Wei and Yi [29] introduced the induced interval-valued intuitionistic fuzzy-ordered weighted geometric (I-IIFOWG) operator, which is defined as follows. 
Definition 2.13 (see [29]). An I-IIFOWG operator of dimension $n$ is a mapping I-IIFOWG: $R^{n} \rightarrow R$ that has an associated vector $w=\left(w_{1}, w_{2}, \ldots, w_{n}\right)^{T}$ such that $\sum_{j=1}^{n} w_{j}=1$ and $w_{j} \in[0,1]$; then

$$
\begin{aligned}
& \operatorname{I-IIFOWG}_{w}\left(\left\langle\varepsilon_{1}, \tilde{\alpha}_{1}\right\rangle,\left\langle\varepsilon_{2}, \tilde{\alpha}_{2}\right\rangle, \ldots,\left\langle\varepsilon_{n}, \tilde{\alpha}_{n}\right\rangle\right)=\tilde{\alpha}_{\pi(1)} w_{1}^{w_{1}} \oplus \tilde{\alpha}_{\pi(2)} w_{2} \oplus \cdots \oplus \tilde{\alpha}_{\pi(n)} w_{n} \\
& =\left(\left[\prod_{j=1}^{n} a_{\pi(j)} w_{j}, \prod_{j=1}^{n} b_{\pi(j)} w_{j}\right],\left[1-\prod_{j=1}^{n}\left(1-c_{\pi(j)}\right)^{w_{j}}, 1-\prod_{j=1}^{n}\left(1-d_{\pi(j)}\right)^{w_{j}}\right]\right),
\end{aligned}
$$

where $\tilde{\alpha}_{\pi(j)}=\left(\left[a_{\pi(j)}, b_{\pi(j)}\right],\left[c_{\pi(j)}, d_{\pi(j)}\right]\right)$ is the $\tilde{\alpha}$ value of the I-IIFOWG pair $\left\langle\varepsilon_{i}, \tilde{\alpha}_{i}\right\rangle$ having the $j$ th largest $\varepsilon_{i}\left(\varepsilon_{i} \in[0,1]\right)$, and $\varepsilon_{i}$ in $\left\langle\varepsilon_{i}, \widetilde{\alpha}_{i}\right\rangle$ is referred to as the order-inducing variable and $\tilde{\alpha}_{i}=\left(\left[a_{i}, b_{i}\right],\left[c_{i}, d_{i}\right]\right)$ as the interval-valued intuitionistic fuzzy values.

In the same way, we have the definition of interval-valued intuitionistic fuzzy-ordered weighted averaging (I-IIFOWA) operator.

Definition 2.14. An I-IIFOWA operator of dimension $n$ is a mapping I-IIFOWA: $R^{n} \rightarrow R$, that has an associated vector with I-IIFOWA operator $w=\left(w_{1}, w_{2}, \ldots, w_{n}\right)^{T}$ such that $\sum_{j=1}^{n} w_{j}=1$ and $w_{j} \in[0,1]$; then

$$
\begin{aligned}
& \operatorname{I-IIFOWA}_{w}\left(\left\langle\varepsilon_{1}, \tilde{\alpha}_{1}\right\rangle,\left\langle\varepsilon_{2}, \tilde{\alpha}_{2}\right\rangle, \ldots,\left\langle\varepsilon_{n}, \tilde{\alpha}_{n}\right\rangle\right)=w_{1} \tilde{\alpha}_{\pi(1)} \oplus w_{2} \tilde{\alpha}_{\pi(2)} \oplus \cdots \oplus w_{n} \tilde{\alpha}_{\pi(n)} \\
& \quad=\left(\left[1-\prod_{j=1}^{n}\left(1-a_{\pi(j)}\right)^{w_{j}}, 1-\prod_{j=1}^{n}\left(1-b_{\pi(j)}\right)^{w_{j}}\right],\left[\prod_{j=1}^{n} c_{\pi(j)} w_{j}, \prod_{j=1}^{n} d_{\pi(j)} w_{j}\right]\right),
\end{aligned}
$$

where $\tilde{\alpha}_{\pi(j)}=\left(\left[a_{\pi(j)}, b_{\pi(j)}\right],\left[c_{\pi(j)}, d_{\pi(j)}\right]\right)$ is the $\tilde{\alpha}$ value of the I-IIFOWA pair $\left\langle\varepsilon_{i}, \tilde{\alpha}_{i}\right\rangle$ having the $j$ th largest $\varepsilon_{i}\left(\varepsilon_{i} \in[0,1]\right)$, and $\varepsilon_{i}$ in $\left\langle\varepsilon_{i}, \tilde{\alpha}_{i}\right\rangle$ is referred to as the order-inducing variable and $\tilde{\alpha}_{i}=\left(\left[a_{i}, b_{i}\right],\left[c_{i}, d_{i}\right]\right)$ as the interval-valued intuitionistic fuzzy values.

$\mathrm{Xu}$ [5] classified all these approaches for determining the weighting vector in aggregation operators into two categories: argument-independent weighting vector method and argument-dependent weighting vector method. The weights derived by the argumentindependent approaches are associated with particular ordered positions of the aggregated arguments and have no connection with the aggregated arguments, while the argumentdependent approaches determine the weights based on the arguments.

In this paper, the argument-weighting vector $\omega=\left(\omega_{1}, \omega_{2}, \ldots, \omega_{n}\right)^{T}$ associated with the inputs $\tilde{\alpha}_{j}$ can be determined by the normal distribution method [13].

Let $\tilde{\alpha}_{1}, \tilde{\alpha}_{2}, \ldots, \tilde{\alpha}_{n}$ be a collection of interval arguments, where $\tilde{\alpha}_{j}=\left(\left[a_{j}, b_{j}\right],\left[c_{j}, d_{j}\right]\right)$, the mean of these interval-valued intuitionistic fuzzy sets $\tilde{u}=\left(\left[a_{\tilde{u}}, b_{\tilde{u}}\right],\left[c_{\tilde{u}}, d_{\tilde{u}}\right]\right)$, where

$$
a_{\tilde{u}}=\frac{1}{n} \sum_{j=1}^{n} a_{j}, \quad b_{\tilde{u}}=\frac{1}{n} \sum_{j=1}^{n} b_{j}, \quad c_{\tilde{u}}=\frac{1}{n} \sum_{j=1}^{n} c_{j}, \quad d_{\tilde{u}}=\frac{1}{n} \sum_{j=1}^{n} d_{j},
$$


and the variance of these interval-valued intuitionistic fuzzy arguments:

$$
\sigma=\sqrt{\frac{1}{n} \sum_{j=1}^{n}\left(d\left(\tilde{\alpha}_{j}, \tilde{u}\right)\right)^{2}} .
$$

then we can have a normal distribution method for deriving the attribute weight associated with its value:

$$
\omega_{j}=\frac{1}{\sqrt{2 \pi} \sigma} e^{-d^{2}\left(\widetilde{\alpha}_{j}-\widetilde{u}\right) / 2 \sigma^{2}}, \quad j=1,2, \ldots, n
$$

Consider that $\omega_{j} \in[0,1]$ and $\sum_{j=1}^{n} \omega_{j}=1$; we have normalized attribute weights:

$$
\omega_{j}=\frac{(1 / \sqrt{2 \pi} \sigma) e^{-d^{2}\left(\tilde{\beta}_{j}-\widetilde{u}\right) / 2 \sigma^{2}}}{\sum_{j=1}^{n}(1 / \sqrt{2 \pi} \sigma) e^{-d^{2}\left(\tilde{\beta}_{j}-\tilde{u}\right) / 2 \sigma^{2}}}, \quad j=1,2, \ldots, n
$$

The position-weighting vector $w=\left(w_{1}, w_{2}, \ldots, w_{n}\right)^{T}$ associated with the operator can be determined by RIM quantifiers [41]:

$$
\begin{gathered}
Q(r)=r^{\alpha}, \quad \alpha \geq 0 \\
w_{i}=Q\left(\frac{i}{n}\right)-Q\left(\frac{i-1}{n}\right), \quad \text { for } i=1,2, \ldots, n
\end{gathered}
$$

\section{Induced Interval-Valued Intuitionistic Fuzzy Hybrid Aggregation Operators with TOPSIS Order-Inducing Variables}

\subsection{I-IIFHA and I-IIFHG Operators}

Induced interval-valued intuitionistic fuzzy hybrid operators are the extensions of ordinary interval-valued intuitionistic fuzzy hybrid operators, and the difference resides in that they can consider more preference information during aggregation process by combination of order-inducing variables independent of arguments. Here, based on the IIFHA and IIFHG, we propose two more general formulations of the reordering process to form I-IIFHA and I-IIFHG operators, which can be defined as follows.

Definition 3.1. An I-IIFHA operator of dimension $n$ is a mapping I-IIFHA: $R^{n} \rightarrow R$ defined by an associated weighting vector $w$ of dimension $n$ such that the sum of the weights is 1 and $w_{j} \in[0,1]$, a set of order-inducing variables $\varepsilon_{j}$, according to the following formula:

$$
\begin{aligned}
& \operatorname{I-IIFHA}_{\omega, w}\left(\left\langle\varepsilon_{1}, \tilde{\alpha}_{1}\right\rangle,\left\langle\varepsilon_{2}, \tilde{\alpha}_{2}\right\rangle, \ldots,\left\langle\varepsilon_{n}, \tilde{\alpha}_{n}\right\rangle\right)=w_{1} \dot{\tilde{\alpha}}_{\pi(1)} \oplus w_{2} \dot{\tilde{\alpha}}_{\pi(2)} \oplus \cdots \oplus w_{n} \dot{\tilde{\alpha}}_{\pi(n)} \\
& \quad=\left(\left[1-\prod_{j=1}^{n}\left(1-\dot{\tilde{a}}_{\pi(j)}\right)^{w_{j}}, 1-\prod_{j=1}^{n}\left(1-\dot{\tilde{b}}_{\pi(j)}\right)^{w_{j}}\right],\left[\prod_{j=1}^{n} \dot{\tilde{\mathcal{C}}}_{\pi(j)} w_{j}, \prod_{j=1}^{n} \dot{\tilde{\alpha}}_{\pi(j)} w_{j}\right]\right),
\end{aligned}
$$


where $\dot{\tilde{\alpha}}_{i}=\left(\left[\dot{\tilde{\alpha}}_{i}, \dot{\tilde{b}}_{i}\right],\left[\dot{\tilde{c}}_{i}, \dot{\tilde{d}}_{i}\right]\right)=n \omega_{i} \widetilde{\alpha}_{i}=\left(\left[1-\left(1-\tilde{a}_{i}\right)^{n \omega_{i}}, 1-\left(1-\widetilde{b}_{i}\right)^{n \omega_{i}}\right],\left[\widetilde{c}_{i}^{n \omega_{i}}, \tilde{d}_{i}^{n \omega_{i}}\right]\right)$, $\left(\dot{\tilde{\alpha}}_{\pi(1)}, \dot{\tilde{\alpha}}_{\pi(2)}, \ldots, \dot{\tilde{\alpha}}_{\pi(n)}\right)$ is $\left(\widetilde{\alpha}_{1}, \tilde{\alpha}_{2}, \ldots, \widetilde{\alpha}_{n}\right)$ reordered in decreasing order of the order-inducing variables values of the $\varepsilon_{j}, \omega=\left(\omega_{1}, \omega_{2}, \ldots, \omega_{n}\right)^{T}$ is the weight vector $\tilde{\alpha}_{j}(j=1,2, \ldots, n)$ with $\sum_{j=1}^{n} \omega_{j}=1$ and $\omega_{j} \in[0,1]$, and $n$ is the balancing coefficient.

Definition 3.2. An I-IIFHG operator of dimension $n$ is a mapping I-IIFHG: $R^{n} \rightarrow R$ defined by an associated weighting vector $w$ of dimension $n$ such that the sum of the weights is 1 and $w_{j} \in[0,1]$, a set of order-inducing variables $\varepsilon_{j}$, according to the following formula:

$$
\begin{aligned}
& \operatorname{I-IIFHG}_{\omega, w}\left(\left\langle\varepsilon_{1}, \tilde{\alpha}_{1}\right\rangle,\left\langle\varepsilon_{2}, \widetilde{\alpha}_{2}\right\rangle, \ldots,\left\langle\varepsilon_{n}, \widetilde{\alpha}_{n}\right\rangle\right) \\
& \quad=\prod_{j=1}^{n} \dot{\tilde{\alpha}}_{\pi(j)} w_{j}=\left(\left[\prod_{j=1}^{n} \dot{\tilde{\alpha}}_{\pi(j)} w_{j}, \prod_{j=1}^{n} \dot{\tilde{b}}_{\pi(j)}^{w_{j}}\right],\left[1-\prod_{j=1}^{n}\left(1-\dot{\tilde{\tilde{c}}}_{\pi(j)}\right)^{w_{j}}, 1-\prod_{j=1}^{n}\left(1-\dot{\tilde{d}}_{\pi(j)}\right)^{w_{j}}\right]\right),
\end{aligned}
$$

where $\dot{\tilde{\alpha}}_{i}=\left(\left[\dot{\tilde{\alpha}}_{i}, \dot{\widetilde{b}}_{i}\right],\left[\dot{\tilde{c}}_{i}, \tilde{\tilde{d}}_{i}\right]\right)=\tilde{\alpha}_{i}{ }^{n \omega_{i}}=\left(\left[a_{i}{ }^{n \omega_{i}}, b_{i}{ }^{n \omega_{i}}\right],\left[1-\left(1-c_{i}\right)^{n \omega_{i}}, 1-\left(1-d_{i}\right)^{n \omega_{i}}\right]\right)$, $\left(\dot{\tilde{\alpha}}_{\pi(1)}, \dot{\tilde{\alpha}}_{\pi(2)}, \ldots, \dot{\tilde{\alpha}}_{\pi(n)}\right)$ is $\left(\tilde{\alpha}_{1}, \tilde{\alpha}_{2}, \ldots, \widetilde{\alpha}_{n}\right)$ reordered in decreasing order of the order-inducing variables values of the $\varepsilon_{j}, \omega=\left(\omega_{1}, \omega_{2}, \ldots, \omega_{n}\right)^{T}$ is the weight vector $\tilde{\alpha}_{i}(i=1,2, \ldots, n)$ with $\sum_{i=1}^{n} \omega_{i}=1$ and $\omega_{i} \in[0,1]$, and $n$ is the balancing coefficient.

Remark 3.3. The argument-weighting vector $\omega=\left(\omega_{1}, \omega_{2}, \ldots, \omega_{n}\right)^{T}$ associated with inputs $\left(\tilde{\alpha}_{1}, \tilde{\alpha}_{2}, \ldots, \widetilde{\alpha}_{n}\right)$ can be determined by normal distribution method defined in (2.17) and (2.18); The position-weighting vector $w=\left(w_{1}, w_{2}, \ldots, w_{n}\right)^{T}$ associated with the operator can be determined by RIM quantifiers in (2.19).

\subsection{TOPSIS Order-Inducing Variables}

Order-inducing variables as reordering step can enable induced aggregation operators to consider additional decision rules that do not depend on the values of the arguments. In this subsection, we propose a TOPSIS-based method to ensure order-inducing variables to be included in I-IIFHA and I-IIFHG operators; as such we can be able to deal with the complex decision-making problems in which the solution with highest score value is not the optimal one for the decision makers.

For example, we have the temperature problem introduced by Marin et al. [25] under linguistic environment. If we are dealing with temperatures of the human body, the optimal term is adequate; if the temperature is higher, the results are not good; and on the other side, if the temperature is lower, the results are also not good. If we are dealing with temperatures at which we want to travel, different persons may be interested in a different ordering of the temperatures; a person may prefer to visit places with cold weather, whereas another person may prefer to warm weather. So proper order-inducing variables should be introduced to deal with the problems where the solution with highest values may not be the optimal one, because of additional decision criteria to influence the ordering of arguments, such as rules of nature and preference of person.

In the induced operators mentioned previously, such as IOWA operator, ILOWA operator, and I-IIFOWA operator, the order-inducing variables have been researched under different environments of exact numbers, interval-valued numbers, or linguistic numbers. 
Here, we let the decision maker define his own preference by interval-valued intuitionistic fuzzy numbers; then a TOPSIS-based method is proposed as follows to derive the orderinducing variables, which is called TOPSIS order-inducing variables.

Step 1. Let the decision maker define the interval-valued intuitionistic fuzzy ideal solution(s) and the interval-valued intuitionistic fuzzy negative ideal solution(s) corresponding to a collection of interval-valued intuitionistic interval arguments $\left(\widetilde{\alpha}_{1}, \widetilde{\alpha}_{2}, \ldots, \widetilde{\alpha}_{n}\right)$ :

$$
\tilde{\alpha}_{j}^{+}=\left(\tilde{\alpha}_{j 1}^{+}, \tilde{\alpha}_{j 2}^{+}, \ldots, \tilde{\alpha}_{j p}^{+}\right), \quad \tilde{\alpha}_{j}^{-}=\left(\tilde{\alpha}_{j 1}^{-}, \tilde{\alpha}_{j 2}^{-}, \ldots, \tilde{\alpha}_{j q}^{-}\right),
$$

where $p(p \geq 0)$ is the number of ideal solution(s), and $q(q \geq 0)$ is the number of negative solution(s).

Step 2. Calculate the distance(s) between the interval-valued intuitionistic interval arguments $\left(\widetilde{\alpha}_{1}, \widetilde{\alpha}_{2}, \ldots, \widetilde{\alpha}_{n}\right)$ and ideal solution(s) and negative solution(s):

$$
d_{j}^{+}=\max _{l=1,2, \ldots, p} d\left(\tilde{\alpha}_{j}, \tilde{\alpha}_{j l}^{+}\right), \quad d_{j}^{-}=\min _{l=1,2, \ldots, q} d\left(\tilde{\alpha}_{j}, \tilde{\alpha}_{j q}^{-}\right),
$$

where $d\left(\widetilde{\alpha}_{j}, \tilde{\alpha}_{j l}^{+}\right)$and $d\left(\widetilde{\alpha}_{j}, \widetilde{\alpha}_{j l}^{-}\right)$can be calculated by the distance measures defined in Definition 2.3. If there is more than one ideal solution, we choose the maximum distance one(s); if there exist more than one negative solution, we choose the minimum distance one(s).

Step 3. Calculate the coefficients

$$
c_{j}=\frac{d_{j}^{-}}{d_{j}^{-}+d_{j}^{+}}, \quad j=1,2, \ldots, n .
$$

Step 4 . Derive the order-inducing variable $u_{j}$ by descending order of $c_{j}$. The greater the value $c_{j}$, the bigger the ordering-induced variable $u_{j}$.

Remark 3.4. In decision-making problems, one decision maker has no interval-valued intuitionistic fuzzy ideal solution(s) or negative solution(s), and we assume that $d_{j}^{+}=1$ and $d_{j}^{-}=0$ if the attribute is the benefit type and $d_{j}^{+}=0$ and $d_{j}^{-}=1$ if it is the cost type.

\subsection{Properties}

Let $f^{A}$ be the I-IIFHA operator, and let $f^{G}$ be the I-IIFHG operator; $\omega=\left(\omega_{1}, \omega_{2}, \ldots, \omega_{n}\right)^{T}$ is argument-weighting vector, and $w=\left(w_{1}, w_{2}, \ldots, w_{n}\right)^{T}$ is position-weighting vector. One has two interval-valued intuitionistic fuzzy vectors:

$$
\begin{gathered}
A=\left\{\left\langle\varepsilon_{\tilde{\alpha}(1)}, \tilde{\alpha}_{1}\right\rangle,\left\langle\varepsilon_{\widetilde{\alpha}(2)}, \tilde{\alpha}_{2}\right\rangle, \ldots,\left\langle\varepsilon_{\widetilde{\alpha}(n)}, \tilde{\alpha}_{n}\right\rangle\right\}, \\
B=\left\{\left\langle\varepsilon_{\widetilde{\beta}(1)}, \tilde{\beta}_{1}\right\rangle,\left\langle\varepsilon_{\widetilde{\beta}(2)}, \widetilde{\beta}_{2}\right\rangle, \ldots,\left\langle\varepsilon_{\widetilde{\beta}(n)}, \tilde{\beta}_{n}\right\rangle\right\} .
\end{gathered}
$$

Then we can get these theorems of I-IIFHA and I-IIFHG operator as follows. 
Theorem 3.5 (monotonicity). The I-IIFHA and I-IIFHG operators are increasingly monotonous with respect to the argument values if the associated order-inducing variables remain unchanged.

$$
\begin{aligned}
& f_{w, \omega}^{A}\left(\left\langle\varepsilon_{\widetilde{\alpha}(1)}, \tilde{\alpha}_{1}\right\rangle,\left\langle\varepsilon_{\widetilde{\alpha}(2)}, \tilde{\alpha}_{2}\right\rangle, \ldots,\left\langle\varepsilon_{\widetilde{\alpha}(n)}, \tilde{\alpha}_{n}\right\rangle\right) \geq f_{w, w}^{A}\left(\left\langle\varepsilon_{\tilde{\beta}(1)}, \tilde{\beta}_{1}\right\rangle,\left\langle\varepsilon_{\tilde{\beta}(2)}, \tilde{\beta}_{2}\right\rangle, \ldots,\left\langle\varepsilon_{\tilde{\beta}(n)}, \tilde{\beta}_{n}\right\rangle\right), \\
& f_{w, \omega}^{G}\left(\left\langle\varepsilon_{\tilde{\alpha}(1)}, \tilde{\alpha}_{1}\right\rangle,\left\langle\varepsilon_{\tilde{\alpha}(2)}, \tilde{\alpha}_{2}\right\rangle, \ldots,\left\langle\varepsilon_{\tilde{\alpha}(n)}, \tilde{\alpha}_{n}\right\rangle\right) \geq f_{w, \omega}^{G}\left(\left\langle\varepsilon_{\tilde{\beta}(1)}, \tilde{\beta}_{1}\right\rangle,\left\langle\varepsilon_{\tilde{\beta}(2)}, \tilde{\beta}_{2}\right\rangle, \ldots,\left\langle\varepsilon_{\tilde{\beta}(n)}, \tilde{\beta}_{n}\right\rangle\right), \\
& \text { if } \tilde{\alpha}_{j} \geq \tilde{\beta}_{j}, \varepsilon_{\tilde{\alpha}(j)}=\varepsilon_{\tilde{\beta}(j)} \forall j \text {. }
\end{aligned}
$$

Proof. Thus, one has $\tilde{\alpha}_{j} \geq \tilde{\beta}_{j} \Rightarrow \dot{\tilde{\alpha}}_{j}=\tilde{\alpha}_{j}{ }^{n \omega_{\tilde{\alpha}(j)}} \geq \dot{\tilde{\beta}}_{j}=\widetilde{\beta}_{j}{ }^{n \omega_{\tilde{\beta}(j)}}$. And $\varepsilon_{\widetilde{\alpha}(j)}=\varepsilon_{\tilde{\beta}(j)}$,

$$
\begin{aligned}
& f_{w, \omega}^{A}\left(\left\langle\varepsilon_{\tilde{\alpha}(1)}, \tilde{\alpha}_{1}\right\rangle,\left\langle\varepsilon_{\tilde{\alpha}(2)}, \tilde{\alpha}_{2}\right\rangle, \ldots,\left\langle\varepsilon_{\tilde{\alpha}(n)}, \tilde{\alpha}_{n}\right\rangle\right) \geq f_{w, \omega}^{A}\left(\left\langle\varepsilon_{\tilde{\beta}(1)}, \tilde{\beta}_{1}\right\rangle,\left\langle\varepsilon_{\tilde{\beta}(2)}, \tilde{\beta}_{2}\right\rangle, \ldots,\left\langle\varepsilon_{\tilde{\beta}(n)}, \tilde{\beta}_{n}\right\rangle\right), \\
& f_{w, \omega}^{G}\left(\left\langle\varepsilon_{\tilde{\alpha}(1)}, \tilde{\alpha}_{1}\right\rangle,\left\langle\varepsilon_{\tilde{\alpha}(2)}, \tilde{\alpha}_{2}\right\rangle, \ldots,\left\langle\varepsilon_{\tilde{\alpha}(n)}, \tilde{\alpha}_{n}\right\rangle\right) \geq f_{w, \omega}^{G}\left(\left\langle\varepsilon_{\tilde{\beta}(1)}, \tilde{\beta}_{1}\right\rangle,\left\langle\varepsilon_{\tilde{\beta}(2)}, \tilde{\beta}_{2}\right\rangle, \ldots,\left\langle\varepsilon_{\tilde{\beta}(n)}, \tilde{\beta}_{n}\right\rangle\right) .
\end{aligned}
$$

Theorem 3.6 (commutativity). The I-IIFHA and I-IIFHG operators are commutative.

$$
\begin{aligned}
& f_{w, \omega}^{A}\left(\left\langle\varepsilon_{\widetilde{\alpha}(1)}, \tilde{\alpha}_{1}\right\rangle,\left\langle\varepsilon_{\widetilde{\alpha}(2)}, \widetilde{\alpha}_{2}\right\rangle, \ldots,\left\langle\varepsilon_{\widetilde{\alpha}(n)}, \tilde{\alpha}_{n}\right\rangle\right)=f_{w, \omega}^{A}\left(\left\langle\varepsilon_{\widetilde{\beta}(1)}, \tilde{\beta}_{1}\right\rangle,\left\langle\varepsilon_{\tilde{\beta}(2)}, \tilde{\beta}_{2}\right\rangle, \ldots,\left\langle\varepsilon_{\widetilde{\beta}(n)}, \tilde{\beta}_{n}\right\rangle\right), \\
& f_{w, \omega}^{G}\left(\left\langle\varepsilon_{\tilde{\alpha}(1)}, \tilde{\alpha}_{1}\right\rangle,\left\langle\varepsilon_{\widetilde{\alpha}(2)}, \tilde{\alpha}_{2}\right\rangle, \ldots,\left\langle\varepsilon_{\tilde{\alpha}(n)}, \tilde{\alpha}_{n}\right\rangle\right)=f_{w, \omega}^{G}\left(\left\langle\varepsilon_{\tilde{\beta}(1)}, \tilde{\beta}_{1}\right\rangle,\left\langle\varepsilon_{\tilde{\beta}(2)}, \tilde{\beta}_{2}\right\rangle, \ldots,\left\langle\varepsilon_{\tilde{\beta}(n)}, \tilde{\beta}_{n}\right\rangle\right),
\end{aligned}
$$

where $\left(\tilde{\alpha}_{1}, \tilde{\alpha}_{2}, \ldots, \tilde{\alpha}_{n}\right)$ is any permutation of the arguments $\left(\tilde{\beta}_{1}, \tilde{\beta}_{2}, \ldots, \tilde{\beta}_{n}\right)$.

Proof. Since $\left(\widetilde{\alpha}_{1}, \widetilde{\alpha}_{2}, \ldots, \widetilde{\alpha}_{n}\right)$ is a permutation of $\left(\widetilde{\beta}_{1}, \widetilde{\beta}_{2}, \ldots, \widetilde{\beta}_{n}\right)$, then we have $\dot{\tilde{\alpha}}_{j}=n \omega_{\widetilde{\alpha}(j)} \widetilde{\alpha}_{j}=$

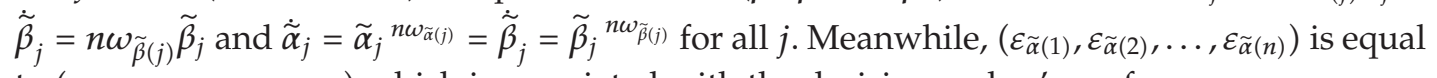
to $\left(\varepsilon_{\tilde{\beta}(1)}, \varepsilon_{\tilde{\beta}(2)}, \ldots, \varepsilon_{\tilde{\beta}(n)}\right)$ which is associated with the decision maker's preference, so

$$
\begin{gathered}
w_{\widetilde{\alpha}(1)} \dot{\tilde{\alpha}}_{1} \oplus w_{\widetilde{\alpha}(2)} \dot{\tilde{\alpha}}_{2} \oplus \cdots \oplus w_{\widetilde{\alpha}(n)} \dot{\tilde{\alpha}}_{n}=w_{\widetilde{\beta}(1)} \dot{\tilde{\beta}}_{1} \oplus w_{\tilde{\beta}(2)} \dot{\tilde{\beta}}_{2} \oplus \cdots \oplus w_{\tilde{\beta}(n)} \dot{\tilde{\beta}}_{n^{\prime}} \\
\dot{\tilde{\alpha}}_{1} w_{\tilde{\alpha}(1)} \otimes \dot{\tilde{\alpha}}_{2} w_{\tilde{\alpha}(2)} \otimes \cdots \otimes \dot{\tilde{\alpha}}_{n} w_{\tilde{\alpha}(n)}=\dot{\tilde{\beta}}_{1} w_{\tilde{\beta}(1)} \otimes \dot{\tilde{\beta}}_{2} w_{\tilde{\beta}(2)} \otimes \cdots \otimes \dot{\tilde{\beta}}_{n} w_{\tilde{\beta}(n)}
\end{gathered}
$$

We get

$$
\begin{aligned}
& f_{w, \omega}^{A}\left(\left\langle\varepsilon_{\widetilde{\alpha}(1)}, \widetilde{\alpha}_{1}\right\rangle,\left\langle\varepsilon_{\widetilde{\alpha}(2)}, \widetilde{\alpha}_{2}\right\rangle, \ldots,\left\langle\varepsilon_{\tilde{\alpha}(n)}, \tilde{\alpha}_{n}\right\rangle\right)=f_{w, \omega}^{A}\left(\left\langle\varepsilon_{\tilde{\beta}(1)}, \tilde{\beta}_{1}\right\rangle,\left\langle\varepsilon_{\tilde{\beta}(2)}, \tilde{\beta}_{2}\right\rangle, \ldots,\left\langle\varepsilon_{\tilde{\beta}(n)}, \tilde{\beta}_{n}\right\rangle\right), \\
& f_{w, \omega}^{G}\left(\left\langle\varepsilon_{\widetilde{\alpha}(1)}, \widetilde{\alpha}_{1}\right\rangle,\left\langle\varepsilon_{\widetilde{\alpha}(2)}, \tilde{\alpha}_{2}\right\rangle, \ldots,\left\langle\varepsilon_{\widetilde{\alpha}(n)}, \tilde{\alpha}_{n}\right\rangle\right)=f_{w, \omega}^{G}\left(\left\langle\varepsilon_{\widetilde{\beta}(1)}, \tilde{\beta}_{1}\right\rangle,\left\langle\varepsilon_{\tilde{\beta}(2)}, \tilde{\beta}_{2}\right\rangle, \ldots,\left\langle\varepsilon_{\widetilde{\beta}(n)}, \tilde{\beta}_{n}\right\rangle\right) .
\end{aligned}
$$


Theorem 3.7 (idempotency). The I-IIFHA and I-IIFHG operators are idempotent. If $\tilde{\alpha}_{j}=\tilde{\alpha}$ for all $j$, then

$$
\begin{aligned}
& f_{w, \omega}^{A}\left(\left\langle\varepsilon_{\tilde{\alpha}(1)}, \tilde{\alpha}_{1}\right\rangle,\left\langle\varepsilon_{\tilde{\alpha}(2)}, \tilde{\alpha}_{2}\right\rangle, \ldots,\left\langle\varepsilon_{\tilde{\alpha}(n)}, \tilde{\alpha}_{n}\right\rangle\right)=\tilde{\alpha}, \\
& f_{w, \omega}^{G}\left(\left\langle\varepsilon_{\tilde{\alpha}(1)}, \tilde{\alpha}_{1}\right\rangle,\left\langle\varepsilon_{\tilde{\alpha}(2)}, \tilde{\alpha}_{2}\right\rangle, \ldots,\left\langle\varepsilon_{\tilde{\alpha}(n)}, \tilde{\alpha}_{n}\right\rangle\right)=\tilde{\alpha} .
\end{aligned}
$$

Proof. Since $\widetilde{\alpha}_{j}=\widetilde{\alpha}$ for all $j$, we have $\left(\omega_{1}, \omega_{2}, \ldots, \omega_{n}\right)=(1 / n, 1 / n, \ldots, 1 / n)$, which is associated with the input arguments $\left(\widetilde{\alpha}_{1}, \tilde{\alpha}_{2}, \ldots, \tilde{\alpha}_{n}\right)$; then

$$
\begin{aligned}
& f_{w, \omega}^{A}\left(\left\langle\varepsilon_{\tilde{\alpha}(1)}, n \omega_{1} \tilde{\alpha}_{1}\right\rangle,\left\langle\varepsilon_{\tilde{\alpha}(2)}, n \omega_{2} \tilde{\alpha}_{2}\right\rangle, \ldots,\left\langle\varepsilon_{\tilde{\alpha}(n)}, n \omega_{n} \tilde{\alpha}_{n}\right\rangle\right) \\
& =f_{w, \omega}^{A}\left(\left\langle\varepsilon_{\tilde{\alpha}(1)}, \tilde{\alpha}_{1}\right\rangle,\left\langle\varepsilon_{\tilde{\alpha}(2)}, \tilde{\alpha}_{2}\right\rangle, \ldots,\left\langle\varepsilon_{\tilde{\alpha}(n)}, \tilde{\alpha}_{n}\right\rangle\right)=w_{1} \tilde{\alpha}_{\pi(1)} \oplus w_{2} \tilde{\alpha}_{\pi(2)} \oplus \cdots \oplus w_{n} \tilde{\alpha}_{\pi(n)} \\
& =\left(w_{1}+w_{2}+\cdots+w_{n}\right) \tilde{\alpha}=\widetilde{\alpha}, f_{w, \omega}^{G}\left(\left\langle\varepsilon_{\tilde{\alpha}(1)}, \tilde{\alpha}_{1}{ }^{n \omega_{1}}\right\rangle,\left\langle\varepsilon_{\widetilde{\alpha}(2)}, \widetilde{\alpha}_{2}{ }^{n \omega_{2}}\right\rangle, \ldots,\left\langle\varepsilon_{\tilde{\alpha}(n)}, \tilde{\alpha}_{n}{ }^{n \omega_{n}}\right\rangle\right) \\
& =f_{w, \omega}^{G}\left(\left\langle\varepsilon_{\tilde{\alpha}(1)}, \tilde{\alpha}_{1}\right\rangle,\left\langle\varepsilon_{\tilde{\alpha}(2)}, \tilde{\alpha}_{2}\right\rangle, \ldots,\left\langle\varepsilon_{\tilde{\alpha}(n)}, \tilde{\alpha}_{n}\right\rangle\right)=\tilde{\alpha}_{\pi(1)} w_{1} \otimes \tilde{\alpha}_{\pi(2)} w_{2} \otimes \cdots \otimes \tilde{\alpha}_{\pi(n)} w_{n} \\
& =\tilde{\alpha}^{\left(w_{1}+w_{2}+\cdots+w_{n}\right)}=\tilde{\alpha} \text {. }
\end{aligned}
$$

Theorem 3.8 (bounded). The I-IIFHA and I-IIFHG operators are bounded. That is, for any weighting vector $\omega$ and w:

$$
\begin{aligned}
& \min \left(\tilde{\alpha}_{1}, \tilde{\alpha}_{2}, \ldots, \tilde{\alpha}_{n}\right) \leq f_{w, \omega}^{A}\left(\left\langle\varepsilon_{\tilde{\alpha}(1)}, \tilde{\alpha}_{1}\right\rangle,\left\langle\varepsilon \tilde{\alpha}(2), \tilde{\alpha}_{2}\right\rangle, \ldots,\left\langle\varepsilon_{\tilde{\alpha}(n)}, \tilde{\alpha}_{n}\right\rangle\right) \leq \max \left(\tilde{\alpha}_{1}, \tilde{\alpha}_{2}, \ldots, \tilde{\alpha}_{n}\right), \\
& \min \left(\widetilde{\alpha}_{1}, \tilde{\alpha}_{2}, \ldots, \tilde{\alpha}_{n}\right) \leq f_{w, \omega}^{G}\left(\left\langle\varepsilon_{\tilde{\alpha}(1)}, \tilde{\alpha}_{1}\right\rangle,\left\langle\varepsilon_{\tilde{\alpha}(2)}, \tilde{\alpha}_{2}\right\rangle, \ldots,\left\langle\varepsilon_{\tilde{\alpha}(n)}, \tilde{\alpha}_{n}\right\rangle\right) \leq \max \left(\widetilde{\alpha}_{1}, \tilde{\alpha}_{2}, \ldots, \tilde{\alpha}_{n}\right) .
\end{aligned}
$$

Proof. By definitions of I-IIFHA and I-IIFHG operators and operations of IVIFNs, we can obtain that

$$
\begin{aligned}
& \min \left\{\tilde{\alpha}_{i}\right\} \leq \min \left\{\tilde{\alpha}_{\pi(i)}\right\} \leq f_{w, \omega}^{A}\left(\left\langle\varepsilon_{\tilde{\alpha}(1)}, \tilde{\alpha}_{1}\right\rangle,\left\langle\varepsilon_{\tilde{\alpha}(2)}, \tilde{\alpha}_{2}\right\rangle, \ldots,\left\langle\varepsilon_{\tilde{\alpha}(n)}, \tilde{\alpha}_{n}\right\rangle\right) \leq \max \left\{\tilde{\alpha}_{\pi(i)}\right\} \leq \max \left\{\tilde{\alpha}_{i}\right\}, \\
& \min \left\{\tilde{\alpha}_{i}\right\} \leq \min \left\{\widetilde{\alpha}_{\pi(i)}\right\} \leq f_{w, \omega}^{G}\left(\left\langle\varepsilon_{\widetilde{\alpha}(1)}, \tilde{\alpha}_{1}\right\rangle,\left\langle\varepsilon_{\widetilde{\alpha}(2)}, \widetilde{\alpha}_{2}\right\rangle, \ldots,\left\langle\varepsilon_{\widetilde{\alpha}(n)}, \widetilde{\alpha}_{n}\right\rangle\right) \leq \max \left\{\tilde{\alpha}_{\pi(i)}\right\} \leq \max \left\{\tilde{\alpha}_{i}\right\} .
\end{aligned}
$$

Theorem 3.9. If $\omega=(1 / n, 1 / n, \ldots, 1 / n)$, I-IIFHA and I-IIFHG operators reduce to I-IIFOWA and I-IIFOWG operators. If $w=(1 / n, 1 / n, \ldots, 1 / n)$, they reduce to IIFWA and IIFWG operators. If there was no any positive or negative preference, $\left(\varepsilon_{\widetilde{\alpha}(1)}, \varepsilon_{\widetilde{\alpha}(2)}, \ldots, \varepsilon_{\tilde{\alpha}(n)}\right)=\left(\varepsilon_{\tilde{\beta}(1)}, \varepsilon_{\tilde{\beta}(2)}, \ldots, \varepsilon_{\tilde{\beta}(n)}\right)$, the IIFHA and IIFHG operators are the special cases of I-IIFHA and I-IIFHG operators.

Proof. Let $\omega=(1 / n, 1 / n, \ldots, 1 / n)$; then

$$
\begin{aligned}
& \operatorname{I-IIFHA}\left(\left\langle\varepsilon_{\widetilde{\alpha}(1)}, n \omega_{1} \tilde{\alpha}_{1}\right\rangle,\left\langle\varepsilon_{\tilde{\alpha}(2)}, n \omega_{2} \tilde{\alpha}_{2}\right\rangle, \ldots,\left\langle\varepsilon_{\widetilde{\alpha}(n)}, n \omega_{n} \tilde{\alpha}_{n}\right\rangle\right) \\
& =w_{1} \tilde{\alpha}_{\pi(1)} \oplus w_{2} \tilde{\alpha}_{\pi(2)} \cdots \oplus w_{n} \tilde{\alpha}_{\pi(n)}=\mathrm{I}-\operatorname{IIFOWA}_{w}\left(\tilde{\alpha}_{1}, \tilde{\alpha}_{2}, \ldots, \tilde{\alpha}_{n}\right), \\
& \operatorname{I-IIFHG}\left(\left\langle\varepsilon_{\tilde{\alpha}(1)}, \tilde{\alpha}_{1}{ }^{n \omega_{1}}\right\rangle,\left\langle\varepsilon_{\widetilde{\alpha}(2)}, \widetilde{\alpha}_{2}{ }^{n \omega_{2}}\right\rangle, \ldots,\left\langle\varepsilon_{\widetilde{\alpha}(n)}, \widetilde{\alpha}_{n}{ }^{n \omega_{n}}\right\rangle\right) \\
& =\tilde{\alpha}_{\pi(1)}{ }^{w_{1}} \otimes \tilde{\alpha}_{\pi(2)}{ }^{w_{2}} \otimes \cdots \otimes \tilde{\alpha}_{\pi(n)}{ }^{w_{n}}=\mathrm{I}-\operatorname{IIFOWG}{ }_{w}\left(\tilde{\alpha}_{1}, \tilde{\alpha}_{2}, \ldots, \tilde{\alpha}_{n}\right) .
\end{aligned}
$$


Let $w=(1 / n, 1 / n, \ldots, 1 / n)$; then

$$
\begin{aligned}
& \operatorname{I-IIFHA}\left(\left\langle\varepsilon_{\tilde{\alpha}(1)}, n \omega_{1} \tilde{\alpha}_{1}\right\rangle,\left\langle\varepsilon_{\tilde{\alpha}(2)}, n \omega_{2} \tilde{\alpha}_{2}\right\rangle, \ldots,\left\langle\varepsilon_{\tilde{\alpha}(n)}, n \omega_{n} \widetilde{\alpha}_{n}\right\rangle\right) \\
& =\omega_{1} \tilde{\alpha}_{1} \oplus \omega_{2} \tilde{\alpha}_{2} \oplus \cdots \oplus \omega_{n} \widetilde{\alpha}_{n}=\operatorname{IIFWA}_{\omega}\left(\widetilde{\alpha}_{1}, \widetilde{\alpha}_{2}, \ldots, \widetilde{\alpha}_{n}\right), \\
& \operatorname{I-IIFHG}\left(\left\langle\varepsilon_{\widetilde{\alpha}(1)}, \widetilde{\alpha}_{1}{ }^{n \omega_{1}}\right\rangle,\left\langle\varepsilon_{\widetilde{\alpha}(2)}, \widetilde{\alpha}_{2}{ }^{n \omega_{2}}\right\rangle, \ldots,\left\langle\varepsilon_{\widetilde{\alpha}(n)}, \widetilde{\alpha}_{n}{ }^{n \omega_{n}}\right\rangle\right) \\
& =\widetilde{\alpha}_{1}{ }^{\omega_{1}} \otimes \widetilde{\alpha}_{2}{ }^{\omega_{2}} \otimes \cdots \otimes \widetilde{\alpha}_{n}{ }^{\omega_{n}}=\operatorname{IIFWG}_{\omega}\left(\widetilde{\alpha}_{1}, \widetilde{\alpha}_{2}, \ldots, \widetilde{\alpha}_{n}\right) \text {. }
\end{aligned}
$$

Theorem 3.10. In the situation of $v_{j}^{L}=0$ or $v_{j}^{L}=0$, interval-valued intuitionistic fuzzy geometric operators are better than interval-valued intuitionistic fuzzy averaging operators, while in $\mu_{j}^{L}=0$ or $\mu_{j}^{U}=0$, interval-valued intuitionistic fuzzy averaging operators are better than interval-valued intuitionistic fuzzy geometric operators.

Proof. Take the IIFWA and IIFWG operators, for example.

$$
\text { If } v_{j}^{L}=0 \text { and } v_{j}^{L}=0 \text {, }
$$

$$
\begin{aligned}
\operatorname{IIFWA}_{\omega}\left(\tilde{\alpha}_{1}, \tilde{\alpha}_{2}, \ldots, \tilde{\alpha}_{n}\right)=\omega_{1} \tilde{\alpha}_{1} \oplus \omega_{2} \tilde{\alpha}_{2} \oplus \cdots \oplus \omega_{n} \tilde{\alpha}_{n} \\
=\left(\left[1-\prod_{j=1}^{n}\left(1-\mu_{j}^{L}\right)^{\omega_{j}}, 1-\prod_{j=1}^{n}\left(1-\mu_{j}^{U}\right)^{\omega_{j}}\right],\left[\prod_{j=1}^{n}\left(v_{j}^{L}\right)^{\omega_{j}}, \prod_{j=1}^{n}\left(v_{j}^{U}\right)^{\omega_{j}}\right]\right) \\
=\left(\left[1-\prod_{j=1}^{n}\left(1-\mu_{j}^{L}\right)^{\omega_{j}}, 1-\prod_{j=1}^{n}\left(1-\mu_{j}^{U}\right)^{\omega_{j}}\right],[0,0]\right) .
\end{aligned}
$$

If $\mu_{j}^{L}=0$ and $\mu_{j}^{U}=0$,

$$
\begin{aligned}
\operatorname{IIFWG}_{\omega}\left(\tilde{\alpha}_{1}, \tilde{\alpha}_{2}, \ldots, \tilde{\alpha}_{n}\right)=\tilde{\alpha}_{1} \omega_{1} \otimes \tilde{\alpha}_{2} \omega_{2} \otimes \cdots \otimes \tilde{\alpha}_{n} \omega_{n} \\
=\left(\left[\prod_{j=1}^{n}\left(\mu_{j}^{L}\right)^{\omega_{j}}, \prod_{j=1}^{n}\left(\mu_{j}^{U}\right)^{\omega_{j}}\right],\left[1-\prod_{j=1}^{n}\left(1-v_{j}^{L}\right)^{\omega_{j}}, 1-\prod_{j=1}^{n}\left(1-v_{j}^{U}\right)^{\omega_{j}}\right]\right) \\
=\left([0,0],\left[1-\prod_{j=1}^{n}\left(1-v_{j}^{L}\right)^{\omega_{j}}, 1-\prod_{j=1}^{n}\left(1-v_{j}^{U}\right)^{\omega_{j}}\right]\right) .
\end{aligned}
$$

Similarly, this rule is also suitable for IIFOWA, IIFOWG, IIFHA, IIFHG, I-IIFOWA, I-IIFOWG, I-IIFHA, I-IIFHG operators, and so forth.

\section{Application in Interval-Valued Intuitionistic Fuzzy Multiple-Attribute Group Decision-Making}

For a multiple-attribute group decision-making problem, let $X=\left\{x_{1}, x_{2}, \ldots, x_{n}\right\}$ be a set of alternatives, and let $G=\left\{g_{1}, g_{2}, \ldots, g_{m}\right\}$ be a set of criteria. Let $D=\left\{d_{1}, d_{2}, \ldots, d_{t}\right\}$ be a set of decision makers, and let $\lambda=\left(\lambda^{(1)}, \lambda^{(2)}, \ldots, \lambda^{(t)}\right)^{T}$ be the weighting vector of decision makers. In the following, we apply the I-IIFHA and I-IIFHG operators (whose positionweighting vector is $w=\left(w_{1}, w_{2}, \ldots, w_{m}\right)^{T}$ to multiple-attribute group decision-making based 
on interval-valued intuitionistic fuzzy information. The approach involves the following steps.

Step 1. Construct the interval-valued intuitionistic fuzzy evaluation matrix.

$\tilde{R}=\left(\tilde{r}_{i j}\right)_{n \times m}=\left(\tilde{\mu}_{i j}, \tilde{v}_{i j}\right)_{n \times m}=\left(\left[\mu_{i j}^{L}, \mu_{i j}^{U}\right],\left[v_{i j}^{L}, v_{i j}^{U}\right]\right)_{n \times m^{\prime}}$, where $\left[\mu_{i j}^{L}, \mu_{i j}^{U}\right]$ indicates the degree at wich the alternative $x_{i}$ satisfies the attribute $g_{j}$ given by the decision maker, and $\left[v_{i j}^{L}, v_{i j}^{U}\right]$ indicates the degree at which the alternative $x_{i}(i=1,2, \ldots, n)$ does not satisfy the attribute $g_{j}(j=1,2, \ldots, m)$.

Step 2. Obtain the interval-valued intuitionistic fuzzy positive-ideal vector and negative-ideal vector corresponding to each alternative's attribute by every decision maker.

Let the $k$ th decision maker provide the interval-valued intuitionistic fuzzy positiveideal vector $\widetilde{R}^{(k)+}$ and the interval-valued intuitionistic fuzzy negative-ideal vector $\widetilde{R}^{(k)-}$

$$
\widetilde{R}^{(k)+}=\left(\tilde{r}_{1}^{(k)+}, \widetilde{r}_{2}^{(k)+}, \ldots, \tilde{r}_{j}^{(k)+}, \ldots, \widetilde{r}_{m}^{(k)+}\right), \quad \widetilde{R}^{(k)-}=\left(\tilde{r}_{1}^{(k)-}, \tilde{r}_{2}^{(k)-}, \ldots, \widetilde{r}_{j}^{(k)-}, \ldots, \widetilde{r}_{m}^{(k)-}\right),
$$

where

$$
\tilde{r}_{j}^{(k)+}=\left(\tilde{r}_{j 1}^{(k)+}, \tilde{r}_{j 2}^{(k)+}, \ldots, \tilde{r}_{j l}^{(k)+}, \ldots, \tilde{r}_{j p}^{(k)+}\right), \quad \tilde{r}_{j}^{(k)-}=\left(\tilde{r}_{j 1}^{(k)-}, \tilde{r}_{j 2}^{(k)-}, \ldots, \tilde{r}_{j l}^{(k)-}, \ldots, \tilde{r}_{j q}^{(k)-}\right) .
$$

Step 3. Calculate the argument-weighting vector $\omega=\left(\omega_{1}, \omega_{2}, \ldots, \omega_{n}\right)^{T}$ associated with the interval-valued intuitionistic fuzzy value $\widetilde{r}_{i j}$ by normal distribution method.

Step 4. Construct aggregated weighted interval-valued intuitionistic fuzzy decision matrix $\dot{\widetilde{R}}=\left(\dot{\widetilde{r}}_{i j}\right)_{n \times m}$ :

$$
\dot{\widetilde{R}}=\left[\begin{array}{ccccc} 
& g_{1} & g_{2} & \cdots & g_{m} \\
x_{1} & \dot{\vec{r}}_{11} & \dot{\vec{r}}_{12} & \cdots & \dot{\widetilde{r}}_{1 m} \\
x_{2} & \dot{\tilde{r}}_{21} & \dot{\vec{r}}_{22} & \cdots & \dot{\tilde{r}}_{2 m} \\
\vdots & \vdots & \vdots & \vdots & \vdots \\
x_{n} & \dot{\vec{r}}_{n 1} & \dot{\vec{r}}_{n 2} & \cdots & \dot{\tilde{r}}_{n m}
\end{array}\right]=\left(\dot{\widetilde{r}}_{i j}\right)_{n \times m^{\prime}}
$$

where $\dot{\tilde{r}}_{i j}=n \omega_{j} \widetilde{r}_{i j}$ or $\dot{\tilde{r}}_{i j}=\widetilde{r}_{i j} n \omega_{j}$, and $\omega=\left(\omega_{1}, \omega_{2}, \ldots, \omega_{n}\right)^{T}$ is the weighting vector associated with interval-valued intuitionistic fuzzy value $\tilde{r}_{i j}$ itself.

Step 5. Ensure the TOPSIS order-inducing variables $\varepsilon$ by using of positive-ideal and negativeideal information provided by each decision maker in Step 2.

First, we calculate the distance between the evaluation information $\tilde{r}_{i j}$ and $k$ th decision maker's preference information.

$$
d_{i j}^{(k)+}=\max _{l=1,2, \ldots, p} d\left(\tilde{r}_{i j}, \tilde{r}_{j l}^{(k)+}\right), \quad d_{i j}^{(k)-}=\min _{l=1,2, \ldots, q} d\left(\tilde{r}_{i j}, \tilde{r}_{j l}^{(k)-}\right)
$$


where $d\left(\widetilde{r}_{i j}, \widetilde{r}_{j}^{(k)+}\right)$ and $d\left(\widetilde{r}_{i j}, \widetilde{r}_{j}^{(k)-}\right)$ can be calculated by the normalized Euclidean distance measure in Definition 2.3.

We can calculate the coefficients of each alternative's attribute:

$$
c_{i j}^{(k)}=\frac{d_{i j}^{(k)-}}{d_{i j}^{(k)-}+d_{i j}^{(k)+}}, \quad i=1,2, \ldots, n, j=1,2, \ldots, m, k=1,2, \ldots, t,
$$

and we can get coefficients matrix $C^{(k)}=\left(c_{i j}^{(k)}\right)_{n \times m}$. By ascending order of $c_{i j}^{(k)}$, we get the ordering induced variables matrix $\varepsilon^{(k)}=\left(\varepsilon_{i j}^{(k)}\right)_{n \times m}$.

Step 6. Utilize the aggregated weighted interval-valued intuitionistic fuzzy decision matrix $\dot{\tilde{R}}=\left(\dot{\tilde{r}}_{i j}\right)_{n \times m}$ and I-IIFHA or I-IIFHG operator to derive the $k$ th decision maker overall interval-valued intuitionistic fuzzy arguments $\widetilde{R}^{(k)}=\left(\widetilde{r}_{1}^{(k)}, \tilde{r}_{2}^{(k)}, \ldots, \tilde{r}_{n}^{(k)}\right)$ corresponding to each alternative $x_{i}$ :

$$
\begin{aligned}
& \tilde{r}_{i}^{(k)}=\left(\left[\mu_{i}^{(k) L}, \mu_{i}^{(k) U}\right],\left[v_{i}^{(k) L}, v_{i}^{(k) U}\right]\right)=\mathrm{I}^{-I_{I F H A}}{ }_{\omega, w}\left(\left\langle\varepsilon_{i 1}^{(k)}, \tilde{r}_{i 1}^{(k)}\right\rangle,\left\langle\varepsilon_{i 2}^{(k)}, \tilde{r}_{i 2}^{(k)}\right\rangle, \ldots,\left\langle\varepsilon_{i m}^{(k)}, \tilde{r}_{i m}^{(k)}\right\rangle\right) \text { or } \\
& \tilde{r}_{i}^{(k)}=\left(\left[\mu_{i}^{(k) L}, \mu_{i}^{(k) U}\right],\left[v_{i}^{(k) L}, v_{i}^{(k) U}\right]\right)=\operatorname{I-IIFHG}_{\omega, w}\left(\left\langle\varepsilon_{i 1}^{(k)}, \tilde{r}_{i 1}^{(k)}\right\rangle,\left\langle\varepsilon_{i 2}^{(k)}, \tilde{r}_{i 2}^{(k)}\right\rangle, \ldots,\left\langle\varepsilon_{i m}^{(k)}, \tilde{r}_{i m}^{(k)}\right\rangle\right) \text {. }
\end{aligned}
$$

Step 7. Utilize the individual overall interval-valued intuitionistic fuzzy arguments vector $\tilde{r}_{i}^{(k)}$ and IIFWA or IIFWG operator to derive the group overall interval-valued intuitionistic fuzzy argument vector $\tilde{r}_{i}$ :

$$
\begin{gathered}
\tilde{r}_{i}=\left(\left[\mu_{i}^{L}, \mu_{i}^{U}\right],\left[v_{i}^{L}, v_{i}^{U}\right]\right)=\operatorname{IIFWA}\left(\tilde{r}_{i}^{(1)}, \tilde{r}_{i}^{(2)}, \ldots, \tilde{r}_{i}^{(t)}\right) \text { or } \\
\tilde{r}_{i}=\left(\left[\mu_{i}^{L}, \mu_{i}^{U}\right],\left[v_{i}^{L}, v_{i}^{U}\right]\right)=\operatorname{IIFWG}\left(\tilde{r}_{i}^{(1)}, \tilde{r}_{i}^{(2)}, \ldots, \tilde{r}_{i}^{(t)}\right) .
\end{gathered}
$$

Step 8. Calculate the scores $s\left(\widetilde{r}_{i}\right)(i=1,2, \ldots, n)$ and the accuracy values $h\left(\widetilde{r}_{i}\right)(i=1,2, \ldots, n)$ of the alternative $x_{i}(i=1,2, \ldots, n)$, respectively.

Step 9. Utilize the scores $s\left(\widetilde{r}_{i}\right)(i=1,2, \ldots, n)$ and the accuracy values $h\left(\tilde{r}_{i}\right)(i=1,2, \ldots, n)$ to rank all the alternatives $x_{i}(i=1,2, \ldots, n)$ and then to select the optimal one(s).

\section{Applied Example}

Here we take the real estate purchase for applied example. There are five candidate houses denoted by $x_{i}(i=1,2,3,4,5)$ that can be evaluated by the IVIFNs $\tilde{r}_{i j}$ with respect to the five attributes: (1) $g_{1}$ : size; (2) $g_{2}$ : price; (3) $g_{3}$ : structure; (4) $g_{4}$ : position; and (5) $g_{5}$ : environment, as listed in Table 1. In the real situation, the DMs may have his/her preference to each attribute. For example, someone may prefer the house close to suburb, while someone may prefer the position of urban area. DMs give their interval-valued intuitionistic fuzzy ideal values and negative ideal values corresponding to five attributes shown in Tables 2, 3, and 4 . Suppose that the expert-weighting vector is $\lambda=(0.3,0.3,0.4)^{T}$. 
Table 1: Decision matrix $\widetilde{R}$ with IVIFNs.

\begin{tabular}{cccccc}
\hline & $g_{1}$ & $g_{2}$ & $g_{3}$ & $g_{4}$ & $g_{5}$ \\
\hline \multirow{2}{*}{$x_{1}$} & $([0.4,0.6]$, & $([0.5,0.7]$, & $([0.5,0.6]$, & $([0.6,0.8]$, & $([0.4,0.7]$, \\
& $[0.3,0.4])$ & $[0.0,0.2])$ & $[0.2,0.4])$ & $[0.1,0.2])$ & $[0.2,0.3])$ \\
$x_{2}$ & $([0.5,0.8]$, & $([0.3,0.5]$, & $([0.3,0.6]$, & $([0.4,0.5]$, & $([0.3,0.6]$, \\
& $[0.1,0.2])$ & $[0.2,0.3])$ & $[0.2,0.4])$ & $[0.2,0.4])$ & $[0.2,0.3])$ \\
$x_{3}$ & $([0.5,0.6]$, & $([0.5,0.8]$, & $([0.4,0.7]$, & $([0.2,0.4]$, & $([0.5,0.8]$, \\
& $[0.0,0.1])$ & $[0.1,0.2])$ & $[0.2,0.3])$ & $[0.2,0.3])$ & $[0.0,0.2])$ \\
$x_{4}$ & $([0.5,0.7]$, & $([0.4,0.6]$, & $([0.3,0.5]$, & $([0.7,0.9]$, & $([0.3,0.5]$, \\
& $[0.1,0.3])$ & $[0.0,0.1])$ & $[0.2,0.4])$ & $[0.0,0.1])$ & $[0.2,0.2])$ \\
$x_{5}$ & $([0.7,0.8]$, & $([0.4,0.6]$, & $([0.4,0.7]$, & $([0.3,0.5]$, & $([0.6,0.7]$, \\
& $[0.0,0.1])$ & $[0.0,0.2])$ & $[0.2,0.3])$ & $[0.1,0.3])$ & $[0.1,0.2])$ \\
\hline
\end{tabular}

Table 2: Preference decision matrix $\widetilde{R}^{(1)}$ provided by $d_{1}$.

\begin{tabular}{lccccc}
\hline$d_{1}$ & $g_{1}$ & $g_{2}$ & $g_{3}$ & $g_{4}$ & $g_{5}$ \\
\hline$\widetilde{R}^{(1)+}([0.5,0.5],[0.5,0.5])$ & $([1,1],[0,0])$ & $([0.5,0.5],[0.5,0.5])$ & $([1,1],[0,0])$ & $([1,1],[0,0])$ \\
$\widetilde{R}^{(1)-}$ & $([0,0],[1,1])$ & $([0,0],[1,1])$ & $([0,0],[1,1])$ & $([1,1],[0,0])$ & $([0,0],[1,1])$ \\
\hline
\end{tabular}

Table 3: Preference decision matrix $\widetilde{R}^{(2)}$ provided by $d_{2}$.

\begin{tabular}{lccccc}
\hline$d_{2}$ & $g_{1}$ & $g_{2}$ & $g_{3}$ & $g_{4}$ & $g_{5}$ \\
\hline$\widetilde{R}^{(2)+}$ & $([1,1],[0,0])$ & $([1,1],[0,0])$ & $([0.3,0.4],[0.6,0.7])$ & $([0.5,0.5],[0.5,0.5])$ & $([1,1],[0,0])$ \\
$\widetilde{R}^{(2)-}$ & $([0,0],[1,1])$ & $([0,0],[1,1])$ & $([1,1],[0,0])$ & $([0,0],[1,1])$ & $([0,0],[1,1])$ \\
\hline
\end{tabular}

Table 4: Preference decision matrix $\widetilde{R}^{(3)}$ provided by $d_{3}$.

\begin{tabular}{lccccc}
\hline$d_{3}$ & $g_{1}$ & $g_{2}$ & $g_{3}$ & $g_{4}$ & $g_{5}$ \\
\hline$\widetilde{R}^{(3)+}$ & $([1,1],[0,0])$ & $([1,1],[0,0])$ & $([0.8,0.9],[0.1,0.2])$ & $([0.5,0.5],[0.5,0.5])$ & $([1,1],[0,0])$ \\
$\widetilde{R}^{(3)-}$ & $([0,0],[1,1])$ & $([0,0],[1,1])$ & $([0,0],[1,1])$ & $([0,0],[1,1])$ & $([0,0],[1,1])$ \\
\hline
\end{tabular}

Table 5: Alternative's attribute coefficient matrix $C^{(1)}$.

\begin{tabular}{lccccc}
\hline$d_{1}$ & $g_{1}$ & $g_{2}$ & $g_{3}$ & $g_{4}$ & $g_{5}$ \\
\hline$x_{1}$ & 0.7513 & 0.6701 & 0.7446 & 0.7321 & 0.6182 \\
$x_{2}$ & 0.6910 & 0.5519 & 0.6734 & 0.5594 & 0.5708 \\
$x_{3}$ & 0.6558 & 0.6910 & 0.7049 & 0.5149 & 0.6865 \\
$x_{4}$ & 0.7011 & 0.6275 & 0.6667 & 0.8000 & 0.5652 \\
$x_{5}$ & 0.6855 & 0.6202 & 0.7049 & 0.5619 & 0.7101 \\
\hline
\end{tabular}

Then we can get the alternative's attribute coefficients matrix $C^{(1)}, C^{(2)}$, and $C^{(3)}$ as shown in Tables 5, 6, and 7 according to (4.4) and (4.5).

By the normal distribution method, we can determine argument-weighting vector associated with their own values:

$$
\omega=(0.2225,0.1911,0.2421,0.1516,0.1927)^{T} .
$$


Table 6: Alternative's attribute coefficient matrix $C^{(2)}$.

\begin{tabular}{cccccc}
\hline$d_{2}$ & $g_{1}$ & $g_{2}$ & $g_{3}$ & $g_{4}$ & $g_{5}$ \\
\hline$x_{1}$ & 0.5658 & 0.6701 & 0.5758 & 0.7061 & 0.6182 \\
$x_{2}$ & 0.6910 & 0.5519 & 0.6073 & 0.7037 & 0.5708 \\
$x_{3}$ & 0.6558 & 0.6910 & 0.5440 & 0.6180 & 0.6865 \\
$x_{4}$ & 0.6569 & 0.6275 & 0.6176 & 0.6813 & 0.5652 \\
$x_{5}$ & 0.7807 & 0.6202 & 0.5440 & 0.6410 & 0.7101 \\
\hline
\end{tabular}

Table 7: Alternative's attribute coefficient matrix $C^{(3)}$.

\begin{tabular}{cccccc}
\hline$d_{3}$ & $g_{1}$ & $g_{2}$ & $g_{3}$ & $g_{4}$ & $g_{5}$ \\
\hline$x_{1}$ & 0.5658 & 0.6701 & 0.7113 & 0.7061 & 0.6182 \\
$x_{2}$ & 0.6910 & 0.5519 & 0.6353 & 0.7037 & 0.5708 \\
$x_{3}$ & 0.6558 & 0.6910 & 0.7112 & 0.6180 & 0.6865 \\
$x_{4}$ & 0.6569 & 0.6275 & 0.6089 & 0.6813 & 0.5652 \\
$x_{5}$ & 0.7807 & 0.6202 & 0.7112 & 0.6410 & 0.7101 \\
\hline
\end{tabular}

Table 8: Aggregate results $\dot{\tilde{R}}$.

\begin{tabular}{cccccc}
\hline & $g_{1}$ & $g_{2}$ & $g_{3}$ & $g_{4}$ & $g_{5}$ \\
\hline \multirow{2}{*}{$x_{1}$} & $([0.3608,0.5665]$, & $([0.5157,0.7112]$, & $([0.4321,0.5388]$, & $([0.6790,0.8444]$, & $([0.4136,0.7092]$, \\
& $[0.3275,0.4335])$ & $[0.0,0.1920])$ & $[0.2367,0.4612])$ & $[0.0768,0.1556])$ & $[0.1935,0.2908])$ \\
$x_{2}$ & $([0.4625,0.7802]$, & $([0.3165,0.5157]$, & $([0.2328,0.5388]$, & $([0.4993,0.5913]$, & $([0.3135,0.6113]$, \\
& $[0.1106,0.2198])$ & $[0.1920,0.2888])$ & $[0.2367,0.4612])$ & $[0.1556,0.3210])$ & $[0.1935,0.2908])$ \\
$x_{3}$ & $([0.4625,0.5665]$, & $([0.5157,0.8080]$, & $([0.3298,0.6494]$, & $([0.2952,0.4993]$, & $([0.5128,0.8065]$, \\
& $[0.0,0.1106])$ & $[0.0958,0.1920])$ & $[0.2367,0.3506])$ & $[0.1556,0.2369])$ & $[0.0,0.1935])$ \\
$x_{4}$ & $([0.4625,0.6725]$, & $([0.4166,0.6138]$, & $([0.2328,0.4321]$, & $([0.7631,0.9232]$, & $([0.3135,0.5128]$, \\
& $[0.1106,0.3275])$ & $[0.0,0.0958])$ & $[0.2367,0.4612])$ & $[0.0,0.0768])$ & $[0.1935,0.1935])$ \\
$x_{5}$ & $([0.6725,0.7802]$, & $([0.4166,0.6138]$, & $([0.3298,0.6494]$, & $([0.4015,0.5913]$, & $([0.6113,0.7092]$, \\
& $[0.0,0.1106])$ & $[0.0,0.1920])$ & $[0.2367,0.3506])$ & $[0.0768,0.2369])$ & $[0.0965,0.1935])$ \\
\hline
\end{tabular}

There exist $v_{x_{i j}}^{L}=0$ and $v_{x_{i j}}^{U}=0$ in interval-valued intuitionistic fuzzy decision matrix $\widetilde{R}$; we choose I-IIFHG and IIFWG operators to aggregate each decision information according to Theorem 3.10 .

We first get the weighted interval-valued intuitionistic fuzzy decision matrix $\dot{\widetilde{R}}=$ $\left(\dot{\widetilde{r}}_{i j}\right)_{n \times m}$ as shown in Table 8 .

Step 6. Suppose the position-weighting vector $w=(0.4472,0.1853,0.1421,0.1198,0.1056)^{T}$ generalized by RIM quantifier. Utilize the aggregated weighted interval-valued intuitionistic fuzzy decision matrix $\dot{\tilde{R}}=\left(\dot{\widetilde{r}}_{i j}\right)_{n \times m}$ and I-IIFHG operator to derive the $k$ th decision maker 
overall interval-valued intuitionistic fuzzy arguments $\widetilde{R}^{(k)}=\left(\widetilde{r}_{1}^{(k)}, \widetilde{r}_{2}^{(k)}, \ldots, \widetilde{r}_{n}^{(k)}\right)$ corresponding to each alternative $x_{i}(i=1,2, \ldots, 5)$.

$$
\begin{array}{ll}
\tilde{r}_{1}^{(1)}=([0.5041,0.6482],[0.1449,0.2952]), & \widetilde{r}_{2}^{(1)}=([0.3410,0.5936],[0.1862,0.3348]), \\
\tilde{r}_{3}^{(1)}=([0.4483,0.6866],[0.0704,0.1991]), & \tilde{r}_{4}^{(1)}=([0.3709,0.5783],[0.1253,0.2871]), \\
\tilde{r}_{5}^{(1)}=([0.4741,0.6772],[0.0806,0.2151]) ; & \\
\tilde{r}_{1}^{(2)}=([0.4583,0.6614],[0.1668,0.3184]), & \tilde{r}_{2}^{(2)}=([0.3311,0.6091],[0.1840,0.3358]), \\
\tilde{r}_{3}^{(2)}=([0.4246,0.6685],[0.1071,0.2202]), & \widetilde{r}_{4}^{(2)}=([0.4439,0.6469],[0.0898,0.1914]), \\
\tilde{r}_{5}^{(2)}=([0.4741,0.6772],[0.0806,0.2151]) ; & \\
\tilde{r}_{1}^{(3)}=([0.4920,0.6994],[0.1344,0.2729]), & \tilde{r}_{2}^{(3)}=([0.3311,0.6091],[0.1840,0.3358]), \\
\tilde{r}_{3}^{(3)}=([0.4483,0.6866],[0.0704,0.1991]), & \tilde{r}_{4}^{(3)}=([0.3858,0.5923],[0.1091,0.2616]), \\
\tilde{r}_{5}^{(3)}=([0.4633,0.6618],[0.1073,0.2351]) . &
\end{array}
$$

Step 7. Use the individual overall interval-valued intuitionistic fuzzy arguments vector $\widetilde{r}_{i}^{(k)}$ and IIFWG operator to derive the group overall interval-valued intuitionistic fuzzy arguments vector $\widetilde{r}_{i}(i=1,2, \ldots, 5)$ :

$$
\begin{array}{ll}
\tilde{r}_{1}=([0.4829,0.6635],[0.1517,0.3002]), & \tilde{r}_{2}=([0.3350,0.6029],[0.1849,0.3354]), \\
\tilde{r}_{3}=([0.4387,0.6793],[0.0853,0.2076]), & \widetilde{r}_{4}=([0.4017,0.6077],[0.1080,0.2450]), \\
\tilde{r}_{5}=([0.4719,0.6741],[0.0860,0.2191]) . &
\end{array}
$$

Step 8. Calculate the scores $s\left(\widetilde{r}_{i}\right)(i=1,2, \ldots, 5)$ of the collective overall intuitionistic fuzzy values by Definition 2.6.

$$
s\left(\widetilde{r}_{1}\right)=0.6945, \quad s\left(\widetilde{r}_{2}\right)=0.4176, \quad s\left(\widetilde{r}_{3}\right)=0.8251, \quad s\left(\widetilde{r}_{4}\right)=0.6564, \quad s\left(\widetilde{r}_{5}\right)=0.8409 .
$$

Step 9. Rank all the alternatives $x_{i}(i=1,2, \ldots, 5)$ in accordance with the scores $s\left(\tilde{r}_{i}\right)(i=$ $1,2, \ldots, 5)$ of the collective overall intuitionistic fuzzy values: $x_{2} \prec x_{4} \prec x_{1} \prec x_{3} \prec x_{5}$, and thus the most desirable alternative is $x_{5}$.

In the previous analysis, the computation of illustrative example is conducted according to the proposed hybrid induced I-IIFHG operator. And we have also conducted computation according to other representative operators: two induced operators of IIIFOWA and I-IIFOWG, which can consider the attitudinal characteristics of experts and the argument-independent preference information; two hybrid operators of IIFHA and IIFHG, which can consider the argument-dependent and argument-independent preference information; and the other proposed induced hybrid I-IIFHA operator. Comparative aggregated results and orderings are listed in Tables 9 and 10. 


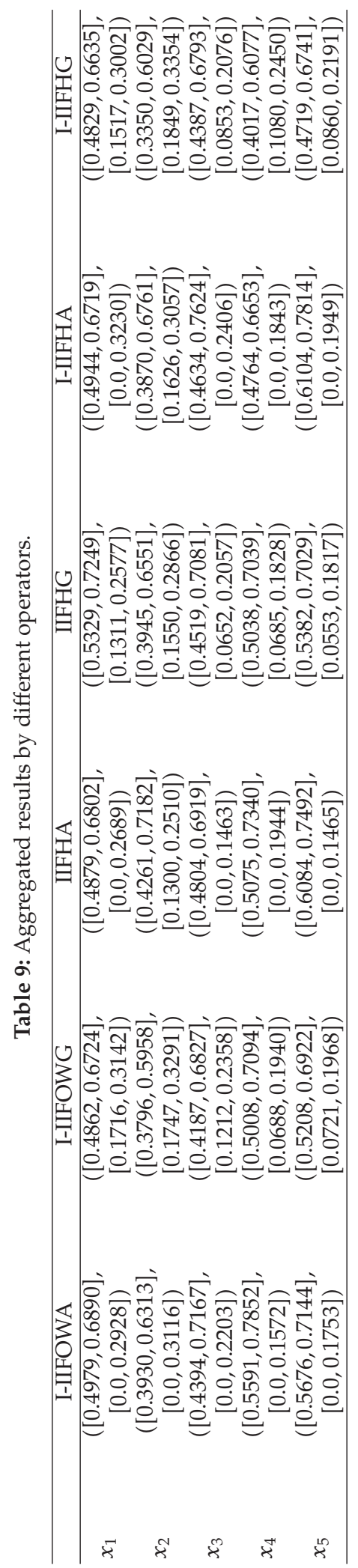


Table 10: Ordering of the candidate houses.

\begin{tabular}{ll}
\hline Different operators & Ordering \\
\hline I-IIFOWA & $2<1<3<5<4$ \\
I-IIFOWG & $2<1<3<5<4$ \\
IIFHA & $2<1<3<4<5$ \\
IIFHG & $2<1<3<4<5$ \\
I-IIFHA & $2<1<4<3<5$ \\
I-IIFHG & $2<4<1<3<5$ \\
\hline
\end{tabular}

As can be seen from the comparative results in Table 10, because the last four hybrid operators (IIFHA, IIFHG, I-IIFHA, and I-IIFHG) manage to give more consideration to the argument-dependent preference information than the first two induced operators (I-IIFOWA and I-IIFOWG), the 5th candidate alternative is emphasized to be the optimal one. And among the last four hybrid operators, because the last two induced hybrid operators (I-IIFHA and I-IIFHG) manage to give more consideration to the additional attitudinal characteristics of experts than the two hybrid operators (IIFHA and IIFHG), the 3rd alternative happens to meet the experts' interests and is emphasized to be better than 1st, 2nd, and 4th alternatives. Also it is can be seen from Table 10, the optimal alternative is consistent with all the last four operators and the worst alternative is consistent with all the six comparative operators.

Generally different aggregation operators for different decision environments may obtain different orderings of candidate solutions. The I-IIFHA and I-IIFHG operators can hybridly aggregate the argument-dependent preference information, argument-independent preference information, and the additional attitudinal characteristics of experts, and can be reduced to different ordinary operators under different decision environments. So the main advantage of the proposed I-IIFHA and I-IIFHG operator with TOPSIS order-inducing variables is that they can include a wide range of particular cases and so can consider a lot of possibilities with a more complete view of the different scenarios when solving multipleattribute multi-interest group decision-making problems under interval-valued intuitionistic fuzzy decision environment.

\section{Conclusion}

We have presented a decision-making approach based on the proposed I-IIFHA and I-IIFHG operators. I-IIFHA and I-IIFHG operators are extensions of the IIFHA and IIFHG operators by using the TOPSIS order-inducing variables in the reordering process. The prominent main advantage of these operators is that they are able to consider more complex attitudinal characteristics in the decision process than other ordinary operators. We have studied some desirable properties of the I-IIFHA and I-IIFHG operators, such as commutativity, monotonicity, idempotency, and bounded. Theoretical analysis has shown that I-IIFHA and I-IIFHG can include a wide range of aggregation operators as special cases, such as IIFHA, IIFOWA, IIFWA, IIFHG, IIFOWG, and IIFWG operators. Furthermore, we have applied these operators to form an approach for the multiple attribute multi-interest group decisionmaking problems with interval-valued intuitionistic fuzzy information. In the future, we will computerize the proposed methods to facilitate the usage and continue working on the extension and application of developed operators to different environments and domains. 


\section{Acknowledgments}

This work was supported by the Social Science Foundation of Ministry of Education of China under Grant 11YJC630283 and Zhejiang Provincial Natural Science Foundation of China under Grant Y6110345.

\section{References}

[1] L. A. Zadeh, "Fuzzy sets," Information and Computation, vol. 8, pp. 338-353, 1965.

[2] K. T. Atanassov, "Intuitionistic fuzzy sets," Fuzzy Sets and Systems, vol. 20, no. 1, pp. 87-96, 1986.

[3] K. Atanassov and G. Gargov, "Interval valued intuitionistic fuzzy sets," Fuzzy Sets and Systems, vol. 31, no. 3, pp. 343-349, 1989.

[4] Z. Xu, "Intuitionistic fuzzy aggregation operators," IEEE Transactions on Fuzzy Systems, vol. 15, no. 6, pp. 1179-1187, 2007.

[5] Z. Xu and R. R. Yager, "Some geometric aggregation operators based on intuitionistic fuzzy sets," International Journal of General Systems, vol. 35, no. 4, pp. 417-433, 2006.

[6] Z. Xu and M. Xia, "Induced generalized intuitionistic fuzzy operators," Knowledge-Based Systems, vol. 24, no. 2, pp. 197-209, 2011.

[7] C. Tan and X. Chen, "Intuitionistic fuzzy Choquet integral operator for multi-criteria decision making," Expert Systems with Applications, vol. 37, no. 1, pp. 149-157, 2010.

[8] Z. Xu and R. R. Yager, "Dynamic intuitionistic fuzzy multi-attribute decison making," International Journal of Approximate Reasoning, vol. 48, no. 1, pp. 246-262, 2008.

[9] G. W. Wei, "Some geometric aggregation functions and their application to dynamic multiple attribute decision making in the intuitionistic fuzzy setting," International Journal of Uncertainty, Fuzziness and Knowledge-Based Systems, vol. 17, no. 2, pp. 179-196, 2009.

[10] Z. S. Xu, "Methods for aggregating interval-valued intuitionistic fuzzy information and their application to decision making," Control and Decision, vol. 22, no. 2, pp. 215-219, 2007.

[11] Z. S. Xu and J. Chen, "An approach to group decision making based on interval-valued intuitionistic judgment matrices," System Engineering Theory and Practice, vol. 27, no. 4, pp. 126-133, 2007.

[12] G. Wei, "Some induced geometric aggregation operators with intuitionistic fuzzy information and their application to group decision making," Applied Soft Computing Journal, vol. 10, no. 2, pp. 423 431, 2010.

[13] Z. Xu, "An overview of methods for determining OWA weights," International Journal of Intelligent Systems, vol. 20, no. 8, pp. 843-865, 2005.

[14] Z. Xu, "Dependent OWA operators," Lecture Notes in Computer Science, vol. 3885, pp. 172-178, 2006.

[15] Z. Xu, "Dependent uncertain ordered weighted aggregation operators," Information Fusion, vol. 9, no. 2, pp. 310-316, 2008.

[16] Z. S. Xu, "Linguistic aggregation operators: an overview," Fuzzy Sets and Their Extensions: Representation, Aggregation and Models, vol. 220, pp. 163-181, 2008.

[17] Z. S. Xu and Q. L. Da, "An overview of operators for aggregating information," International Journal of Intelligent Systems, vol. 18, no. 9, pp. 953-969, 2003.

[18] C. Y. Liang, J. Wu, and W. X. Lu, "A new method on hybrid multiple attribute decision-making problem for choosing the supplier," Chinese Journal of Management Science, vol. 14, pp. 71-76, 2006.

[19] P. D. Liu, "A novel method for hybrid multiple attribute decision making," Knowledge-Based Systems, vol. 22, no. 5, pp. 388-391, 2009.

[20] J. M. Merigó and M. Casanovas, "The induced generalized hybrid averaging operator and its application in financial decision making," IWorld Academy of Science, Engineering and Technology, vol. 55, pp. 1065-1071, 2009.

[21] J. M. Merigó and M. Casanovas, "Fuzzy generalized hybrid aggregation operators and its application in fuzzy decision making," International Journal of Fuzzy Systems, vol. 12, no. 1, pp. 15-24, 2010.

[22] G.-W. Wei, "Uncertain linguistic hybrid geometric mean operator and its application to group decision making under uncertain linguistic environment," International Journal of Uncertainty, Fuzziness and Knowledge-Based Systems, vol. 17, no. 2, pp. 251-267, 2009.

[23] G. W. Wei and W. D. Yi, "Uncertain linguistic hybrid geometric mean operator," in Proceedings of the 4th International Conference on Fuzzy Systems and Knowledge Discovery (FSKD '07), pp. 497-501, Haikou, China, August 2007. 
[24] F. Chiclana, E. Herrera-Viedma, F. Herrera, and S. Alonso, "Some induced ordered weighted averaging operators and their use for solving group decision-making problems based on fuzzy preference relations," European Journal of Operational Research, vol. 182, no. 1, pp. 383-399, 2007.

[25] L. Marin, J. M. Merigó, A. Valls, A. Moreno, and D. Isern, "Induced unbalanced linguistic ordered weighted average," Advances in Intelligent Systems Research, vol. 1, pp. 1-8, 2011.

[26] J. M. Merigó and M. Casanovas, "Induced aggregation operators in decision making with the Dempster-Shafer belief structure," International Journal of Intelligent Systems, vol. 24, no. 8, pp. 934-954, 2009.

[27] J. M. Merigó and M. Casanovas, "Induced aggregation operators in the Euclidean distance and its application in financial decision making," Expert Systems with Applications, vol. 38, no. 6, pp. 76037608, 2011.

[28] J. Wu, Q. W. Cao, and J. L. Zhang, "Some properties of the induced continuous ordered weighted geometric operators in group decision making," Computers and Industrial Engineering, vol. 59, no. 1, pp. 100-106, 2010.

[29] G. Wei and W. Yi, "Induced interval-valued intuitionistic fuzzy OWG operator," in Proceedings of the 5th International Conference on Fuzzy Systems and Knowledge Discovery (FSKD '08), pp. 605-609, Shandong, China, October 2008.

[30] Z. Xu, "Induced uncertain linguistic OWA operators applied to group decision making," Information Fusion, vol. 7, no. 2, pp. 231-238, 2006.

[31] Z. Xu, "On generalized induced linguistic aggregation operators," International Journal of General Systems, vol. 35, no. 1, pp. 17-28, 2006.

[32] R. R. Yager, “Induced aggregation operators," Fuzzy Sets and Systems, vol. 137, no. 1, pp. 59-69, 2003.

[33] J. M. Merigó and M. Casanovas, "Induced and uncertain heavy OWA operators," Computers and Industrial Engineering, vol. 60, no. 1, pp. 106-116, 2011.

[34] J. M. Merigó and A. M. Gil-Lafuente, "The induced generalized OWA operator," Information Sciences, vol. 179, no. 6, pp. 729-741, 2009.

[35] J. M. Merigó and A. M. Gil-Lafuente, "Fuzzy induced generalized aggregation operators and its application in multi-person decision making," Expert Systems with Applications, vol. 38, no. 8, pp. 9761-9772, 2011.

[36] J. M. Merigó, A. M. Gil-Lafuente, L. G. Zhou, and H. Y. Chen, “Induced andlinguistic generalized aggregation operators and their application in linguistic group decision making," Group Decision and Negotiation. In press.

[37] Z. X. Su and G. P. Xia, "Induced generalized intuitionistic fuzzy OWA operator for multi-attribute group decision making," Expert Systems with Applications, vol. 39, pp. 1902-1910, 2012.

[38] C. Tan and X. Chen, "Induced intuitionistic fuzzy Choquet integral operator for multicriteria decision making," International Journal of Intelligent Systems, vol. 26, no. 7, pp. 659-686, 2011.

[39] J. M. Merigó and M. Casanovas, "Induced and heavy aggregation operators with distance measures," Journal of Systems Engineering and Electronics, vol. 21, no. 3, pp. 431-439, 2010.

[40] J. M. Merigó and M. Casanovas, "Decision-making with distance measures and induced aggregation operators," Computers and Industrial Engineering, vol. 60, no. 1, pp. 66-76, 2011.

[41] R. R. Yager, "Quantifier guided aggregation using OWA operators," International Journal of Intelligent Systems, vol. 11, no. 1, pp. 49-73, 1996. 


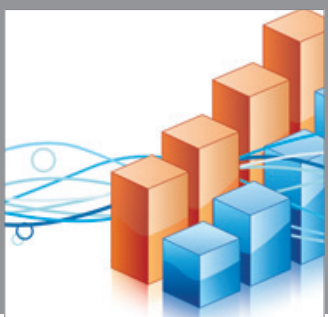

Advances in

Operations Research

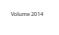

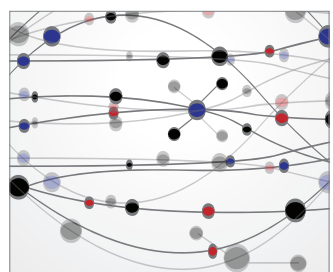

\section{The Scientific} World Journal
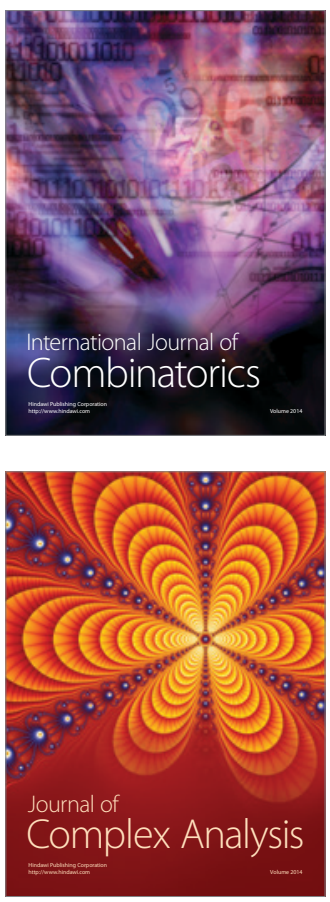

International Journal of

Mathematics and

Mathematical

Sciences
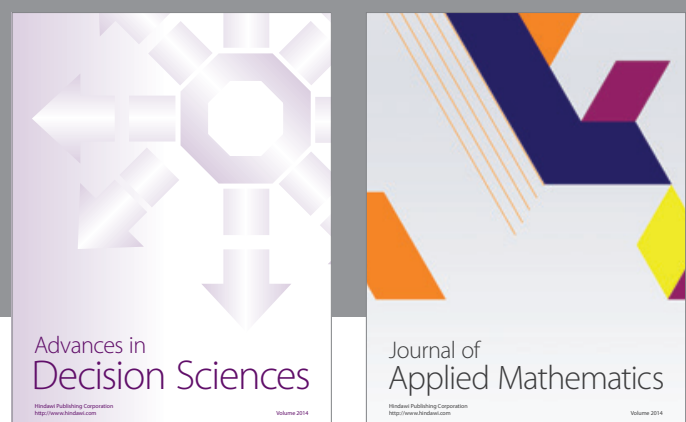

Journal of

Applied Mathematics
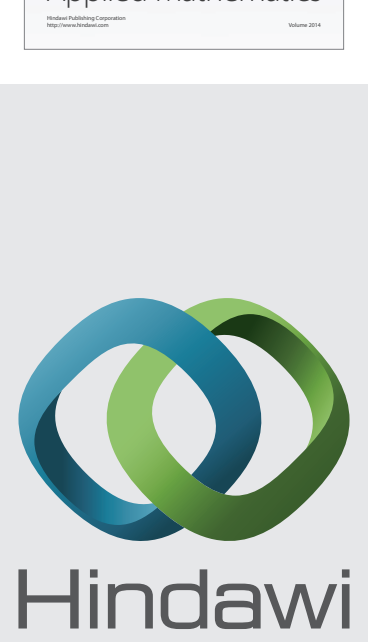

Submit your manuscripts at http://www.hindawi.com
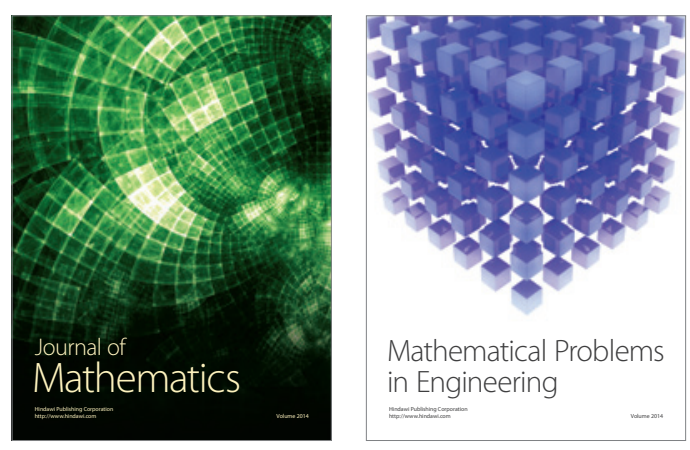

Mathematical Problems in Engineering
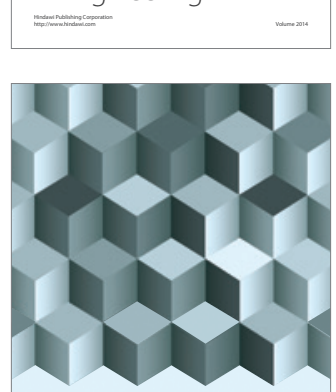

Journal of

Function Spaces
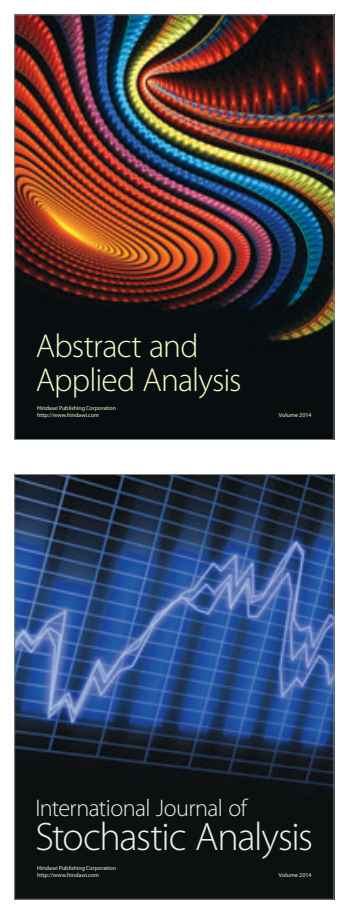

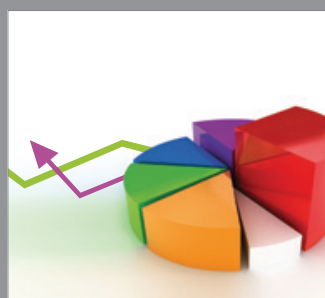

ournal of

Probability and Statistics

Promensencen
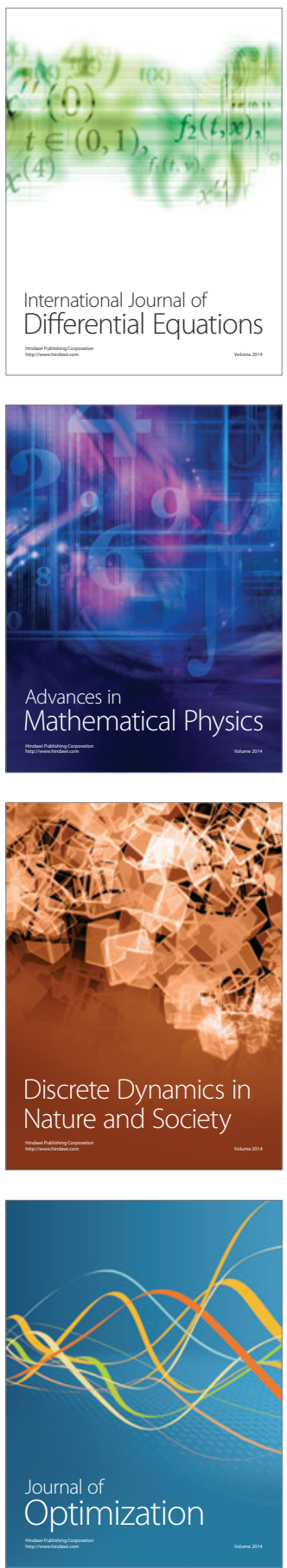